\title{
South African guideline for the management of community-acquired pneumonia in adults
}

\author{
Tom H. Boyles ', Adrian Brink' ${ }^{1,2}$, Greg L. Calligaro ${ }^{3}$, Cheryl Cohen ${ }^{4,5}$, Keertan Dheda ${ }^{3}$, Gary Maartens ${ }^{6}$, Guy A. Richards ${ }^{7}$, \\ Richard van Zyl Smit ${ }^{3}$, Clifford Smith ${ }^{8}$, Sean Wasserman ${ }^{1}$, Andrew C. Whitelaw ${ }^{9,10}$, Charles Feldman ${ }^{11}$; \\ South African Thoracic Society, Federation of Infectious Diseases Societies of Southern Africa
}

'Division of Infectious Diseases and HIV Medicine, Department of Medicine, University of Cape Town, Cape Town, South Africa;

${ }^{2}$ Ampath National Laboratory Services, Milpark Hospital, Johannesburg, South Africa;

${ }^{3}$ Lung Infection and Immunity Unit, Division of Pulmonology and UCT Lung Institute, University of Cape Town, Cape Town, South Africa;

${ }^{4}$ Centre for Respiratory Disease and Meningitis, National Institute for Communicable Diseases, Johannesburg, South Africa;

${ }^{5}$ School of Public Health, University of the Witwatersrand, Johannesburg, South Africa;

${ }^{6}$ Division of Clinical Pharmacology, Department of Medicine, University of Cape Town, Cape Town, South Africa;

${ }^{7}$ Department of Critical Care, Faculty of Health Sciences, University of the Witwatersrand, Johannesburg, South Africa;

${ }^{8}$ Morningside Mediclinic, Sandton, South Africa;

${ }^{9}$ Division of Medical Microbiology, Faculty of Medicine and Health Sciences, Stellenbosch University, Stellenbosch, South Africa;

${ }^{10}$ National Health Laboratory Service, Tygerberg Hospital, Cape Town, South Africa;

${ }^{11}$ Charlotte Maxeke Johannesburg Academic Hospital and Faculty of Health Sciences, University of the Witwatersrand, Johannesburg,

South Africa.

Correspondence to: Charles Feldman, email: Charles.feldman@wits.ac.za.

Republished with permission from Journal of Thoracic Disease. 2017;9(6):1469-1502

\section{Executive summary}

Improving the care of patients with community-acquired pneumonia (CAP) in South Africa is particularly important because of the high burden of disease and the need to improve standards of antibiotic prescribing in the face of rising antimicrobial resistance (AMR). The purpose of this document is to provide clinicians guidance as to the recommended management of patients with CAP. This is an update for clinicians, which takes into account important advances and controversies in the management of patients with CAP.

\section{Diagnosing CAP}

\section{Primary care}

The definitive clinical diagnosis of pneumonia requires the presence of compatible symptoms and signs for $<2$ weeks plus a new or worsening consolidation on chest X-ray (CXR). CXR may not be available in primary care settings in which case the diagnosis can be made on clinical grounds alone.

- CAP should be diagnosed in patients in primary care who present with a combination of well-established clinical features of CAP, including vital sign and examination abnormalities (A II).

\section{Hospital level care}

In contrast to primary care, CXRs are widely available and all patients presenting to hospital with suspected CAP require a CXR to confirm the diagnosis and exclude other potential causes for their illness. Otherwise the principles of CAP diagnosis are the same as in primary care.
- A CXR should be performed in all patients presenting to hospital with suspected CAP (A II).

- In the vast majority of cases a normal CXR excludes the diagnosis of CAP; however, empiric antibiotic therapy can be considered for severely ill hospitalised patients with suspected CAP and a negative CXR study. CAP is excluded if a repeat CXR at 24-48 hours is negative (A III).

\section{Severity of illness scores}

Assessment of the severity of CAP is important since it will determine the appropriate site of care, the extent of the microbiological work-up and the choice of initial empiric antibiotic treatment.

- The CURB-65 score (CRB-65 for outpatients) is the recommended disease severity score for patients with CAP (A II).

- Severity scoring systems should not be the sole basis for making decisions regarding site of care. Disease severity score should always be interpreted in conjunction with a thorough clinical assessment of the patient (A II).

\section{Site of care decisions}

Site of care decisions, such as outpatient vs. inpatient care or general ward vs. intensive care unit, are important areas for improvement of CAP care. Decisions should be based on the clinical condition of the patients, on the disease severity scoring, on the social circumstances of the patients and on available resources.

- Patients with a CRB-65 score of 0 or a CURB-65 score of 0 or 1 are at low risk of death and may be considered for treatment at home (A II). 
- Patients with a CRB-65 score of 1 or 2 or a CURB-65 score of 2 are at increased risk of death, and should be referred to hospital (A II).

- Patients with a CRB-65 score or CURB-65 score of 3 or more are at high risk of death and require urgent hospital admission and even consideration for possible admission to a high-care or intensive care unit (A II).

\section{Additional tests}

\section{Blood-based biomarkers}

Blood-based biomarkers may be used to aid the diagnosis of CAP and to assist in severity assessment.

- Routine measurement of CRP or PCT when the diagnosis is not in doubt is discouraged but may be used to measure response to therapy in the critically ill (A III).

- Measurement of CRP, particularly in primary care settings and when CXR is unavailable, may aid the diagnosis of CAP (A II).

- Measurement of CRP or PCT in emergency departments may be considered in patients with acute respiratory illness when the diagnosis of CAP is in doubt (B II).

- Urea should be measured in all hospitalised patients with CAP to assist in severity scoring (A I).

\section{Microbiological tests}

- Blood cultures (BCs) should be taken prior to antibiotic therapy in all patients with CAP with a CURB-65 score of $\geq 2$ (A II).

- BCs should be considered in patients with lower CURB-65 scores, but who require hospitalisation for other reasons (B II).

- BCs should not be performed on patients with CAP who are being treated as outpatients (A II).

- A sputum sample or tracheal aspirate (collected at intubation) should be submitted for Gram stain and culture for all patients with CAP with a CURB-65 score of $\geq 2$ (A II).

- Sputum samples can be considered in patients with CURB-65 scores of $<2$ who require hospitalisation for reasons such as comorbidities (B II).

- Sputum samples should not be submitted on patients with CAP who are being treated as outpatients (A II).

- The use of the pneumococcal UAT is not routinely recommended for patients with CAP (B II).

- The Legionella UAT should be considered, where available, for patients with severe CAP (B III).

- The use of rapid antigen tests for influenza is not recommended (B II).

- In patients with severe CAP during the influenza season (typically June to September) nasopharyngeal samples may be considered for detection of influenza (B II).

- The routine use of molecular tests to detect additional pathogens is not recommended (B II).

- Serology for 'atypical' pathogens should not be routinely performed (A II).

\section{Investigating for tuberculosis}

TB is a cause of CAP and clinical features are not reliable in distinguishing TB from other aetiologies. However, TB should be suspected in patients presenting with CAP who are co-infected with HIV, have a subacute history and in those who initially do not respond to antibiotics. Specific investigations for TB should be performed as indicated.

- In the following high risk patient groups presenting with CAP there should be a low threshold for investigation for pulmonary TB: HIV-infected, diabetics, admission to ICU, subacute illness or those not responding to empiric antimicrobial therapy (A II).

- A GeneXpert MTB/RIF ${ }^{T M}$ (Cepheid, Sunnyvale, USA) assay performed on a single expectorated or induced sputum specimen is the preferred first line diagnostic test for pulmonary TB. Alternatively, WHO endorsed rapid molecular tests, such as line probe assays, are recommended when they are more readily available (A II).

- TB culture should be performed in the following patients with a negative Gene Xpert MTB/RIF: non-resolving pneumonia or an ongoing suspicion of TB (A III).

- When sputum is unavailable Determine ${ }^{\mathrm{TM}}$ TB-LAM Ag (Alere, Waltham, MA, USA) testing should be performed in HIVinfected patients with CD4 counts $<100$ cells/ $\mu$ l or stage 3 or 4 disease who present with CAP (A I).

\section{Investigating for pneumocystis pneumonia}

PCP typically presents in immunocompromised patients as a subacute illness with constitutional symptoms and dry cough, and is characterised by bilateral infiltrates on CXR.

- The WHO clinical case definition should be used to clinically diagnose PCP (B III).

- Diagnostic testing of HIV-infected patients who fit the WHO case definition or in whom PCP is suspected on clinical grounds depends on local availability of tests and may include an immunofluorescent assay (IFA), direct fluorescent antibody test (DFAT) or PCR (B III).

- The preferred specimen for diagnostic tests for PCP is bronchoalveolar lavage fluid (BAL) although induced or expectorated sputum may be used when bronchoscopy is unavailable (B II).

- There is limited evidence to support for the use of beta-glucan to diagnose PCP in a South African setting. Its use is only recommended as part of a clinical registry or trial (A III).

\section{Initial empiric therapy}

Initial empiric therapy for CAP should be guided by the setting in which the patient is being treated, their age, use of antibiotics within the previous 90 days, the presence of comorbidities (cardiovascular disease, chronic respiratory disease, chronic renal failure, diabetes mellitus and HIV infection) and drug intolerance. Empiric therapy for PCP and influenza may be necessary when clinical and epidemiological criteria are met. It is rarely necessary to give empiric treatment for TB unless there is a miliary pattern on CXR. 


\section{Initial antibiotic therapy}

- Patients treated at home who are $<65$ years old, without antibiotic exposure in the past 90 days or comorbidities should receive oral high dose amoxicillin (A II).

- Patients treated at home who are $<65$ years old, without antibiotic exposure in the past 90 days, or comorbidities, in the setting of low macrolide resistance, could receive an oral macrolide/azalide in the presence of severe beta-lactam allergy (A II).

- Patients treated at home who are $\geq 65$ years old, have received antibiotics within the previous 90 days, or who have comorbidities, should receive oral amoxicillin-clavulanate or an oral second generation cephalosporin (A II).

- Patients whose admission to hospital is precipitated by advanced age, personal or family preference, inadequate home care or adverse social circumstances who have nonsevere pneumonia, can be treated with oral antibiotics as described above (A II).

- Patients requiring admission to hospital who are $<65$ years old, without antibiotic exposure in the past 90 days, or comorbidities, should receive intravenous ampicillin or penicillin (if IVI ampicillin not available) (A II).

- Patients requiring admission to hospital who are $\geq 65$ years old, have received antibiotics within the previous 90 days, or who have comorbidities, should receive intravenous amoxicillinclavulanate, or cefuroxime or a third-generation cephalosporin (ceftriaxone or cefotaxime) (A II).

- Patients with severe pneumonia should receive amoxicillinclavulanate or cefuroxime or a third generation cephalosporin (ceftriaxone or cefotaxime) plus a macrolide antibiotic (A II).

- Respiratory fluoroquinolones (moxifloxacin or levofloxacin) are an alternative therapy but because of their activity against tuberculosis these agents should not be used as first line in CAP. They may be used in patients with severe beta-lactam allergy or as an alternative to beta-lactam/macrolide therapy but should be reserved for use in patients who have no alternative treatment options (A II).

- Antibiotics should be administered early, preferably within the emergency unit, to patients with confirmed CAP (A II).

\section{Definitive therapy}

When microbiological testing detects a causative organism, it may be possible to change from empiric to definitive therapy based on the drug susceptibility testing.

- When a causative organism is identified by microbiological testing, antibiotics should be changed to the narrowest spectrum agent that effectively treats the organism (A II).

- Ceftaroline is recommended as directed therapy based on the results of microbiological testing in cases of high level penicillin resistant (penicillin MIC $\geq 8 \mathrm{mg} / \mathrm{L}$ ) S. pneumoniae or MRSA (A I).
- Ertapenem is recommended as directed therapy based on the results of microbiological testing in cases of resistant Enterobacteriaceae such as ESBL-producing pathogens (A I).

\section{When to add therapy for PCP and TB}

- Empiric therapy for PCP should be added when patients fulfill the WHO case definition and it should not be withheld based on negative immunohistochemical staining on sputum specimens (A II).

- Empiric therapy for TB prior to initial testing is rarely required unless there is a miliary pattern on CXR or the patient is severely ill and TB is suspected (A III).

\section{When to add empiric therapy for influenza}

- During the influenza season oseltamivir should be provided for any patient with severe pneumonia and can be stopped if PCR testing of nasopharyngeal aspirate is negative (A II).

- During the influenza season oseltamivir should be provided for any patient with moderate CAP who is suspected of having influenza if they have a specific risk factor for severe disease and can be stopped if PCR testing of nasopharyngeal aspirate is negative ( $\mathrm{B} I \mathrm{I})$.

\section{Adjunctive therapies}

Given the significant burden of disease caused by CAP there have been many attempts to find adjunctive therapies to improve outcomes.

- There is not enough evidence to recommend the routine use of statins for either prevention or treatment of CAP (A II).

- The addition of a macrolide to standard beta-lactam therapy is associated with a better outcome in patients with severe CAP requiring $I C U$ admission and while this may relate to the antimicrobial activity of macrolides, it may also be due to their anti-inflammatory, immunomodulatory effects (A II).

- Use of systemic corticosteroids (e.g., methylprednisone $0.5 \mathrm{mg} / \mathrm{kg} / 12 \mathrm{~h}$ or equivalent) should be considered in patients with severe CAP requiring ICU admission unless influenza or tuberculosis is likely, or there is a history of gastro-intestinal bleeding within the previous 3 months (A I).

\section{Intravenous to oral switching (IVPOS) and duration of antibiotics}

Prompt switching from intravenous to oral therapy is a cornerstone of antibiotic stewardship, as is the use of the minimum effective duration of therapy.

- Patients can switch from intravenous to oral antibiotics when they are haemodynamically stable, have a respiratory rate $<25 /$ min, temperature $<37.8{ }^{\circ} \mathrm{C}$ and are able to take oral medication (A I).

- For patients managed in the community, and for most patients admitted to hospital with low or moderate severity and uncomplicated pneumonia, 5-7 days of appropriate antibiotics is recommended (A II). 
- Treatment duration may be extended beyond 14 days for specific clinical scenarios such as Staphylococcus aureus bacteraemia (A II).

- Patients with confirmed Legionella pneumonia should be treated with azithromycin for 7 days (A II).

\section{Acute complications}

A number of possible complications of CAP may occur and are recognised in patients who fail to respond to the first few days of empiric therapy or who deteriorate after an initial improvement.

\section{Complicated para-pneumonic effusion and empyema}

- Repeat CXR should be performed for any patient failing to respond to the first few days of empiric therapy or who deteriorates after an initial improvement (A II).

- If follow-up CXR demonstrates effusion or lung abscess, further imaging with $\mathrm{CT}$ or thoracic ultrasonography should be considered (B II).

- Any significant amount of pleural fluid should prompt diagnostic pleurocentesis to exclude empyema (A II).

- Fluid drainage by mean of an intercostal drain is necessary in all cases of complicated para-pneumonic effusion or empyema (A II).

\section{Lung abscess}

- Patients diagnosed with lung abscess as a complication of CAP should receive a prolonged course of antibiotics, usually 4-6 weeks, along with physiotherapy to effect postural drainage (B II).

\section{Cardiovascular events}

- Patients with CAP with well-recognised risk factors for a cardiovascular event or cases that fail to show adequate clinical recovery should be investigated for the possibility of a cardiovascular event (A III).

\section{Aspiration pneumonia}

The term 'aspiration' refers to the abnormal entry of a large inoculum of exogenous substances or endogenous secretions into the lower airways. This can cause pneumonitis or pneumonia, which has important clinical and microbiological differences from CAP.

- Acute aspiration events, particularly in the absence of systemic inflammation or impaired respiratory function, do not require antimicrobial therapy, even if associated with a new CXR infiltrate (A III).

- Antimicrobials should be considered for patients with aspiration pneumonitis and persistent or progressive signs and symptoms 48 hours after aspirating (B III).

- Aspiration pneumonia may be a more indolent process, usually occurring late after the aspiration event, and may be associated with suppurative complications. The diagnosis implies bacterial infection of the lung, and is therefore an indication for antimicrobial therapy (A II).
- Recommended empiric antibiotic therapy is amoxicillinclavulanate; a cephalosporin plus clindamycin or metronidazole may be an acceptable alternative (B II).

\section{Vaccination for prevention of pneumonia}

Appropriate vaccination, typically against Streptococcus pneumoniae and influenza virus is a key pillar of antibiotic stewardship.

- All adults $\geq 50$ years who are vaccine naive should receive a single dose of PCV13 (A II).

- All adults $\geq 50$ years who have received PPV23 should receive a single dose of PCV13 one year later (A II).

- All adults $\geq 65$ years who are vaccine naive should receive a single dose of PCV13 followed a year later by PPV23 (A II).

- All adults $\geq 65$ years who have received PPV23 should receive a single dose of PCV13 at least one year later (A II).

- Younger adults ( $\geq 18$ years) who are vaccine naïve with severe underlying comorbid or immunocompromising conditions including HIV infection should receive a single dose of PCV13 followed at least 2 months later by PPV23 (A II).

- Younger adults ( $\geq 18$ years) who have previously received PPV23 and have severe underlying comorbid or immunocompromising conditions including HIV infection should receive a single dose of PCV13 one year later (A II).

- All women who are pregnant in the period of influenza vaccine availability (approximately March to June) should be offered influenza vaccination with IIV3 (All). Adults aged $\geq 65$ years should be offered annual influenza vaccination with IIV3 (A I).

- All adults with specific chronic diseases (chronic pulmonary [including tuberculosis] and cardiac diseases, chronic renal diseases, diabetes mellitus and similar metabolic disorders, individuals who are immunosuppressed including HIVinfected individuals, and individuals who are morbidly obese (body mass index $\left.\geq 40 \mathrm{~kg} / \mathrm{m}^{2}\right)$ ) should be offered annual influenza vaccination with IIV3 (A II).

- All healthcare workers should be offered annual influenza vaccination with IIV3 (A II).

\section{Introduction}

CAP causes considerable morbidity and mortality throughout the world (1-3). Improving the care of patients with CAP in South Africa is particularly important because of the high burden of disease and the need to improve standards of antibiotic prescribing in the face of rising AMR (4).

There are a number of important international guidelines including those of the Infectious Diseases Society of America (IDSA)/American Thoracic Society (ATS) (5) and the British Thoracic Society (BTS) (6). However, South Africa represents a unique environment with a high prevalence of both HIV infection and AMR such that guidelines must be locally applicable. Groups interested in approaches to the management of CAP in South Africa include the South African Thoracic Society (SATS) and the 
Federation of Infectious Diseases Societies of Southern Africa (FIDSSA). This guideline, under the auspices of SATS and FIDSSA, represents an update of the SATS CAP Guideline published in 2007 (7).

Box 1: Strength of recommendations

Strength of recommendation
A: strong recommendation for or against use
B: moderate recommendation for or against use
C: weak recommendation for or against use
Quality of evidence
I: evidence from at least 1 properly randomised, controlled trial
II: evidence from at least 1 well-designed clinical trial without
randomisation, from cohort or case-controlled analytic studies
(preferably from more than 1 centre), from multiple time-series, or
from dramatic results from uncontrolled experiments
III: evidence from opinions of respected authorities, based on clinical
experience, descriptive studies, or reports of expert committees

\section{Purpose and scope}

The purpose of this document is to update clinicians regarding important advances and controversies in the management of patients with CAP. South Africa has a high prevalence of HIV infection, which is a risk factor for a number of pulmonary infections with overlapping presentations including tuberculosis and Pneumocystis jirovecii pneumonia (PCP). This guideline seeks to provide practical advice on the approach to all adult patients with acute community-acquired infection of the lung parenchyma. These guidelines do not apply to the much larger group of adults with non-pneumonic lower respiratory tract infections (LRTI), including acute bronchitis, acute exacerbations of chronic obstructive pulmonary disease or illnesses labelled as 'chest infections'. Pneumonia in non-ambulatory residents of nursing homes and other long-term care facilities epidemiologically mirrors healthcare-associated pneumonia (HCAP) and should be treated according to institutional HCAP guidelines.

\section{Methodology}

A committee of specialists from the SATS and the FIDSSA was assembled with individuals and/or small groups being assigned to write a section of the guideline. A non-systematic literature search was undertaken to identify published evidence relevant to each section. Searches were conducted in PubMed and The Cochrane Library databases, as well as by checking reference lists of highly relevant papers and recent international CAP guidelines. Sections were collated and distributed to the entire group for comment. Disagreements were resolved by online discussion. Once all comments were addressed the committee agreed to the final draft of the guideline. Attempts have been made to align the guideline with the Department of Health Essential Medicines List, however it is aimed at both public and private sectors and therefore differs in some respects. The committee recognises that most patients with CAP are cared for by primary care, internal medicine, and emergency medicine physicians, and this guideline is therefore directed primarily at them.

\section{Grading of recommendations}

The strength of recommendations in this guideline is illustrated in Box 1.

\section{Epidemiology}

Influenza and pneumonia, along with tuberculosis were amongst the top five leading underlying natural causes of death in South African adults aged $\geq 15$ years in 2013 (8). The incidence of LRTI amongst individuals aged $\geq 15$ years in South Africa is approximately 400 per 100,000 populations with the peak incidence in individuals aged 25-64 years, likely driven by the high HIV prevalence in this age group (9). Underlying HIV infection is the most important risk condition for LRTI hospitalisation in South Africa, with an HIV prevalence of $74 \%$ among hospitalised patients aged $\geq 5$ years (and $>90 \%$ among those aged $25-44$ years) documented at 4 sentinel surveillance sites in South Africa between 2009-2012 (9). HIV-infected individuals have substantially greater risk of being hospitalised with LRTI than HIV-uninfected individuals. Other important risk factors for hospitalised LRTI in adults include increasing age and underlying lung disease such as asthma or chronic obstructive airways disease. In South Africa from 2009-2012, the case fatality ratio (CFR) in adults with hospitalised LRTI was $7 \%$ with HIV-infected individuals experiencing a higher CFR (8\%) than HIV-uninfected individuals (5\%) (9). Other significant predictors of mortality in this setting were increasing age-group and receiving mechanical ventilation or supplemental oxygen.

Many organisms can cause pneumonia in adults. Mixed infections with multiple viral and/or bacterial infections are common. Streptococcus pneumoniae is the most common cause of CAP [approximately $27 \%$ of all adult CAP in the pre-pneumococcal conjugate vaccine (PCV) era] (10), although the incidence of invasive pneumococcal pneumonia in South African adults has decreased as a result of indirect effect following the introduction of PCV into the routine infant immunisation programme in 2009 (11). Other bacterial causes of pneumonia include Haemophilus influenzae, Staphylococcus aureus and gram-negative bacilli (such as Klebsiella pneumoniae). Atypical bacterial causes of pneumonia are uncommon in South Africa ( $<2 \%$ of all adult (AP) but they have cyclical circulation with periodic increases in incidence and some (e.g., Legionella and Mycoplasma species) may cause outbreaks (12). Bordetella pertussis may present as CAP more atypically in older children and adults. In 2014, pertussis was identified in $<5 \%$ of all patients with LRTI, however, rates of pertussis in all ages may be expected to increase following the change from whole-cell to acellular pertussis vaccine in the routine infant immunisation schedule in 2009. Anaerobic bacteria may cause pneumonia particularly in patients at increased risk of aspiration. Pseudomonas aeruginosa is another uncommon bacterial cause of CAP and may be considered in individuals with severe pneumonia, especially those with 
underlying malignancy or cardiovascular disease and structural lung disease e.g., cystic fibrosis (13).

Influenza is the most common viral cause of CAP in adults, identified in $9 \%$ of patients. Other respiratory viruses such as respiratory syncytial virus, rhinovirus and adenovirus may also be identified (14). Co-infection is common with respiratory viruses and bacteria and some viruses may also be identified from healthy controls with no respiratory symptoms; therefore, identification of a virus should not preclude initiation of antibiotic treatment.

Importantly, between $18 \%$ to $40 \%$ of patients with CAP in South Africa may test positive for tuberculosis $(15,16)$. The spectrum of aetiological agents in HIV-infected individuals may differ from HIV-uninfected individuals with Streptococcus pneumoniae and tuberculosis being more commonly identified in HIV-infected individuals (9). In addition, it is important to consider the diagnosis of PCP which has a prevalence of $22 \%$ among HIV-infected adults admitted with CAP in sub-Saharan Africa $(17,18)$. P. jirovecii was identified on quantitative PCR from $18 \%$ of adult CAP patients in South Africa in 2014; however in some cases, identification on PCR may indicate colonisation (12). The risk of CAP in HIV-infected individuals, as well as the probability of identifying mixed or atypical infections, increases with decreasing CD4+T cell count (19).

With increasing globalisation and increasing contact at the animal-human interface, the risks of emerging respiratory viral infections such as avian influenza or Middle East Respiratory Syndrome Coronavirus (MERS-CoV) infections remain present (20). Clinicians should keep themselves updated regarding global trends in emerging viruses. Clusters of two or more individuals hospitalised with severe respiratory illness or respiratory deaths with no identified aetiology should be investigated.

\section{Diagnosing CAP}

\section{Primary care}

Pneumonia refers to an infection of the lower respiratory tract resulting in parenchymal lung inflammation and symptoms of an acute illness. The clinical diagnosis of pneumonia requires the presence of compatible symptoms and signs plus a new or worsening infiltrate on CXR (21). In this guideline patients with 'suspected CAP' will be defined as having supportive clinical features (described below) without CXR confirmation.

Although cough is the most common reason for presentation to outpatient care (22), only a minority of these patients will have CAP $(6,23,24)$. Other LRTIs, including acute bronchitis, are most commonly caused by viruses $(25,26)$, are associated with normal chest radiographs, and do not require antibiotic therapy $(27,28)$. In a recent randomised controlled trial involving over 2,000 outpatients with LRTI in whom pneumonia was not suspected, the group assigned amoxicillin had no benefit in terms of duration or severity of symptoms. Side effects were significantly more common in those receiving antibiotics compared to placebo, translating into a net harmful effect (27). Similar findings have been observed in other clinical trials (29-31), emphasising the need to correctly identify those with CAP to limit overuse of antibiotics and to appropriately treat and refer those with more serious infections.

Without the benefit of CXR the diagnosis of CAP in outpatient settings is challenging and relies on clinical finding (6) and, if available, point-of-care (POC) biomarkers (32). A suggested way of diagnosing CAP in primary care when CXR is unavailable using clinical features and CRP has recently been presented (33). The accuracy of clinical criteria for diagnosing CAP is poor; a systematic review has shown that even combinations of symptoms and signs rarely increase the probability of the diagnosis by more than $50 \%$ (34). However, the absence of vital sign abnormalities is a relatively reliable way to exclude the diagnosis (35), and one study found that patients presenting with LRTI and normal vital signs and clinical examination have a low risk of pneumonia, and that this could be used to rule out the diagnosis in $95 \%$ of cases without the use of CXR (36).

Although there is a poor correlation between clinical findings and radiologically confirmed CAP, most physicians in primary care rely on history and examination to exclude or diagnose this disease (35). The following features are most commonly associated with $\operatorname{CAP}(6,29,37,38)$ and should be sought in all patients presenting with acute cough to primary care:

Symptoms of an acute LRTI-cough, pleuritic chest pain, shortness of breath, sputum production (and the absence of rhinorrhoea or sore throat) (24).

New focal chest signs on examination-localised bronchial breathing or crackles, dullness on percussion, decreased chest expansion.

Systemic abnormalities—temperature $\geq 38^{\circ} \mathrm{C}$, subjective fever or chills, sweating, tachycardia.

No other explanation for the illness-acute aspiration, carcinoma, pulmonary embolism, pulmonary oedema, acute exacerbation of COPD.

Pneumonia is a leading cause of morbidity and mortality in the elderly (39-41), who are at increased risk for this disease because of immunosenescence and concomitant illness (42). Atypical presentations are more common (43), leading to delays in diagnosis and treatment (44). Fever and cough are frequently absent in frail older adults, in whom CAP may manifest as new or worsening confusion, deterioration in functional status or decompensation of an underlying illness (44-47). In additional to these non-specific clinical findings, the elderly more commonly have normal CXRs (48), making the diagnosis especially challenging.

\section{Recommendations}

- CAP should be diagnosed in patients in primary care who present with a combination of well-established clinical 
features of CAP, including vital sign and examination abnormalities (A II).

\section{Hospital level care}

The principles of CAP diagnosis in patients presenting to hospital are the same as for outpatients. In contrast to primary care, X-rays are widely available and all patients presenting to hospital with suspected CAP require a CXR to confirm the diagnosis and exclude other potential causes for their illness (5).

The accuracy of CXR for the diagnosis of pneumonia is not known but it is considered the reference standard for rulingout the diagnosis of CAP when normal. However, even a normal CXR is not sufficient to eliminate the diagnosis in the setting of a very high pre-test probability (34). CXR is not a good test for ruling-in CAP and alternative diagnoses should be considered for patients with abnormal CXRs in the absence of compatible clinical features of CAP. Therefore, as in all infectious diseases, the diagnosis of CAP in severely ill patients depends on the likelihood (or risk) of having the disease and the finding of supportive features using a combination of history, examination and chest radiography. In severely ill hospitalised patients with suspected pneumonia and a normal CXR, it is not unreasonable to start empiric antibiotic therapy (48), which may be stopped if appropriate cultures are negative and a repeat CXR at 24-48 hours remains clear of new infiltrates (5). This is supported by a large cohort study where $7 \%$ of patients admitted with suspected CAP and an initially normal CXR developed changes consistent with CAP on a repeat CXR (48). In most circumstances, however, the administration of antibiotics for suspected CAP without CXR confirmation has been associated with inaccurate diagnosis (49) and overuse of antibiotics (50).

\section{Recommendations}

- A CXR should be performed in all patients presenting to hospital with suspected CAP (A II).

- In the vast majority of cases a normal CXR excludes the diagnosis of CAP; however, empiric antibiotic therapy can be considered for severely ill hospitalised patients with suspected CAP and a negative CXR study. CAP is excluded if a repeat CXR at $24-48$ hours is negative (A III).

\section{Severity of illness scores}

A number of assessment tools have been developed to assist clinicians in assessing the severity of CAP with each having its own advantages and limitations (51). However, assessment of the severity of infection is important since it will determine the appropriate site of care, the extent of the microbiological workup and the choice of initial, empiric antibiotic treatment. One such scoring system recommended for use in patients admitted to hospital is the CURB-65 score, which was derived from the BTS rules. The original international derivation and validation study of the CURB-65 was published in 2003 (52), and although it has also been validated in other settings, it has not been formally validated in the South African setting, as is the case with most of these scoring systems. The CURB-65 scoring system uses 5 components namely:

- Confusion.

- Urea $>7 \mathrm{mmol} / \mathrm{L}$.

- Respiratory rate $\geq 30$ breaths/min.

- Low blood pressure (systolic $<90 \mathrm{mmHg}$ and/or diastolic $\leq 60 \mathrm{mmHg}$ ).

- Age $\geq 65$ years.

A point is assigned for each of the parameters, if present in the patient. This scoring system has been recommended for use because of its simplicity, but also because its accuracy is similar to that of more complicated scoring systems, such as the pneumonia severity index (PSI).

A variation of the CURB-65 is the CRB-65, which does not require the measurement of the serum urea, thus making it particularly suitable for outpatient use, although it may be a little less accurate. The potential accuracy of the CRB-65 score in determining the need for hospitalisation of patients with CAP in an emergency department of a teaching hospital in Johannesburg, a setting of resource constraints and high HIV infection prevalence, has been evaluated. This was a prospective observational study in a single centre (53), which, although not an interventional study, documented that the CRB-65 accurately predicted time to clinical stability and risk of death in the patients with CAP. It appeared from the investigation that if the CRB-65 were to have been implemented in that setting it would have performed well in decision-making regarding the need for hospital admission of the patients. None of the scoring systems can replace clinical assessment and important additional factors, such as socioeconomic deprivation and comorbidity, must also influence the decision on hospitalisation and treatment.

\section{Recommendations}

- The CURB-65 score (CRB-65 for outpatients) is the recommended disease severity score for patients with CAP (A II).

- Severity scoring systems should not be the sole basis for making decisions regarding site of care. Disease severity score should always be interpreted in conjunction with a thorough clinical assessment of the patient (A II).

\section{Site of care decisions}

Patients may be referred from primary care to hospital for confirmation of the diagnosis with additional tests such as CXR or because admission is warranted. For patients seen in emergency departments with confirmed CAP a decision must be made regarding the need for admission. These decisions should be based on a combination of objective severity scoring, clinical assessment and factors such as advanced age, presence of significant co-morbid conditions such as HIV infection and COPD, and social circumstances. In general, patients seen in primary care with a CRB-65 score of 0 should be considered 
for treatment at home and those with scores of $\geq 1$ should be considered for referral for admission to hospital.

The decision to admit patients seen in emergency departments with confirmed CAP should be based on the CURB-65 score supplemented with a subjective clinical assessment. It is preferable to manage patients with CAP outside of hospital (54), because of the increased costs $(55,56)$ and complication rates associated with hospital admission. Patients with a CURB65 score of 0 and 1 are thought to have a mild infection and are potentially suitable for management at home. Patients with scores of 2 are considered moderately ill and need to be observed in hospital, at least initially. Patients with scores of 3 or more are thought to be severely ill and therefore these cases, and especially those with a score of 4 or 5, need evaluation for possible admission to a high-care or even intensive care unit.

\section{Recommendations}

- Patients with a CRB-65 score of 0 or a CURB-65 score of 0 or 1 are at low risk of death and may be considered for treatment at home (B II).

- Patients with a CRB-65 score of 1 or 2 or a CURB-65 score of 2 are at increased risk of death, and should be referred to hospital (A II).

- Patients with a CRB-65 or CURB-65 score of 3 or more are at high risk of death and require urgent hospital admission and even consideration for admission to a high-care or intensive care unit (A II).

\section{Additional tests}

\section{Blood markers}

CRP can be useful when the diagnosis of CAP is in doubt (57), although primary care settings are less likely to have the test available. One exception is point-of-care CRP machines that are available in some primary healthcare facilities, particularly in the private sector. A systematic review and meta-analysis of CRP use in patients with respiratory tract infection presenting to primary care facilities showed a significant reduction in antibiotic use with a suggestion that this may lead to slightly more hospital admissions (32).

If CAP is confirmed by clinical presentation and CXR the value of CRP is very limited as it is raised in almost all cases (58). There are conflicting data regarding its use as a prognostic marker and it is a poor discriminator between different aetiologies of CAP and between TB and CAP. CRP is a sensitive marker of response to therapy with a failure to fall by $50 \%$ at day 3 or 4 being associated with increased 30-day mortality (59). In practice, however, clinical evaluation, including serial measurement of temperature, is likely to be sufficient to identify patients requiring further investigation and/or a change in antibiotic therapy.

The use of procalcitonin to guide initiation and discontinuation of antibiotics in patients with a range of respiratory tract infections across a range of settings has shown a significant reduction in the use of antibiotics with no increase in mortality or treatment failure (60). However, sub-group analysis of patients with CAP shows little difference in the proportion of patients initially prescribed antibiotics. It is therefore not recommended that procalcitonin be measured when the diagnosis of CAP is confirmed. Procalcitonin is expensive and routine measurement to guide duration of antibiotics is not recommended.

Full blood count (FBC) is of limited diagnostic value in CAP. While a raised white cell count (WCC) implicates a bacterial aetiology this is not excluded if the count is normal. FBC is of more value when the diagnosis is in doubt and alternative diagnoses are being sought. Routine measurement of urea in patients with CAP is important as it forms part of the CURB-65 severity score. Routine measurement of liver function is not recommended.

\section{Recommendations}

- Routine measurement of CRP or PCT when the diagnosis is not in doubt is discouraged but may be used to measure response to therapy in the critically ill (A III).

- Measurement of CRP, particularly in primary care settings and when CXR is unavailable, may aid the diagnosis of CAP (A II).

- Measurement of CRP or PCT in emergency departments may be considered in patients with acute respiratory illness when the diagnosis of CAP is in doubt (B II).

- Urea should be measured in all hospitalised patients with CAP to assist severity scoring (A I).

\section{Microbiological investigations}

Microbiological investigations are performed in order to identify the aetiological agent, and are not intended to confirm the diagnosis of CAP, which is diagnosed primarily clinically and radiologically. The common microbiological investigations that would be performed in the setting of CAP are BC, sputum culture, and detection of Legionella and pneumococcal antigens in urine. In addition, molecular tests are becoming more commonly employed, and serology may also be used. The benefit of identifying the aetiological agent is primarily to assist in directing antimicrobial therapy. This can result either in a change to broaden the spectrum of activity for an organism not adequately treated by the initial, empiric, regimen (which would directly benefit the patient), or to change to a narrower spectrum agent if a susceptible organism is identified (which is less likely to directly benefit the patient, but is in line with antibiotic stewardship principles, and will hopefully have a broader benefit on society). Other reasons to attempt to identify aetiological agents are to identify organisms of potential public health importance (e.g., influenza) and to better understand the epidemiology of CAP-which in turn influences clinical practice. However, these investigations are associated with increased health care costs. The decision of whether or not to perform microbiological investigations in patients with CAP is sometimes debateable. As a general rule, investigations would be recommended where there is a reasonable chance of the result influencing the management of the patient. 
The yield of $B C$ in the setting of CAP ranges from about 5-14\%. Guidelines for appropriate collection of BCs have been published (61), and will not be reviewed here. The likelihood of a patient with CAP having a positive BC is increased with severity of illness. While a number of studies have described clinical parameters associated with increased chance of a positive $B C$, due to differences in study design and clinical parameters described, different studies have identified different parameters associated with bacteraemia. These include neutrophilia (62), low WCC, raised creatinine, hypoglycaemia, temperature $>38^{\circ} \mathrm{C}(63)$, elevated PSI score (64); tachycardia, tachypnoea and hypotension (65), and low oxygen saturation (66).

The use of sputum to identify aetiological agents is bedevilled by the need for an adequate sputum sample. When adequate samples are collected (as assessed by the presence of neutrophils and absence of epithelial cells), the Gram stain may be positive in up to $82 \%$ of patients with bacteremic pneumococcal pneumonia, $76 \%$ of patients with bacteremic Staphylococcus aureus pneumonia and $78 \%$ of patients with gram-negative bacteraemia (67). Thus, the major benefit of a Gram stain on a well-collected sputum sample is that it may allow for the broadening of antimicrobial cover for S. aureus or gram-negative bacilli. However, it must be borne in mind that in the majority of patients, the microscopy will be unhelpful, with no predominant micro-organism observed. Only approximately $25-40 \%$ of patients will have an adequate sample obtained $(68,69)$ and the yield on culture of these adequate samples can be as high as $80 \%$. However, when assessing the sputum culture yield in all patients with CAP, it can be as low as $15 \%$ overall, since a proportion of patients are unable to produce sputum, and a proportion of samples will be unsatisfactory. As with BCs, sputum samples should only be sent for culture if the result is likely to influence patient management.

The two commercially available urine antigen tests (UATs) are for Legionella pneumophila serogroup 1, and for Streptococcus pneumoniae. The Legionella antigen test has a high specificity (99\%), but sensitivity is not well defined. The pneumococcal antigen test has a sensitivity of $60-80 \%$, and specificity of about $90 \%(70,71)$.

The major issue with both of these tests is whether positive results will significantly alter therapy. In addition, for the pneumococcal antigen test, the question is what incremental yield is obtained over sputum and BC. A recent meta-analysis found that the UAT may identify an additional $11 \%$ of patients with pneumococcal pneumonia, over and above those identified with culture (10). However, a study from Switzerland found that the addition of the UAT for $S$. pneumoniae did not influence antibiotic choices or clinical outcomes (72). Given that empiric regimens for CAP always include agents active against $S$. pneumoniae this is not surprising. A similar argument could be made regarding the use of the Legionella UAT, since patients with severe CAP will be treated with an agent active against this pathogen. However, there are no readily available alternative tests to detect Legionella, and the knowledge may have epidemiological importance. Thus, while further research is needed to guide practice regarding narrowing of spectrum when a UAT is positive, there may be some justification for testing patients with severe CAP for Legionella using a UAT.

Rapid antigen detection tests (RADT) are also available for influenza. Earlier versions of these assays had relatively poor sensitivity (50-70\%), and although more recent versions have improved sensitivity there have been concerns that they are still not as sensitive as molecular assays (73). There is also little evidence to date on the clinical impact of using RADT in preference to molecular assays for influenza.

The addition of molecular tests to the diagnostic armamentarium has significantly increased the proportion of patients in whom an aetiological agent can be found-up to $80 \%(74,75)$. The majority of these additional agents have been viral, although molecular tests do offer the ability to detect some of the socalled 'atypical' pathogens. The challenge, however, remains in determining whether the presence of a positive molecular result proves causality, since the same viruses may be found in a proportion of asymptomatic patients. In addition, for many of the viral pathogens there is no specific treatment (apart from influenza).

The exception to the above discussion is influenza. Specific treatment is available, and the detection of influenza has epidemiologic and public health implications. Molecular assays also offer the most rapid and reliable method to detect influenza at present, although rapid antigen tests may prove superior and more cost effective in future.

Themajordisadvantage of serologicalinvestigation forpathogens such as Legionella, Chlamydophila and Mycoplasma spp., is the need for acute and convalescent sera to allow for appropriate interpretation of results. Thus, the results will not impact immediate patient management, and the routine use of serology is not recommended.

\section{Recommendations}

- BCs should be taken prior to antibiotic therapy in all patients with CAP with a CURB-65 score of $\geq 2$ (A II).

- BCs should be considered in patients with lower CURB-65 scores, but who require hospitalisation for other reasons (B II).

- BCs should not be performed on patients with CAP who are being treated as outpatients (A II).

- A sputum sample or tracheal aspirate (collected at intubation) should be submitted for Gram stain and culture for all patients with CAP with a CURB-65 score of $\geq 2$ (A II).

- Sputum samples can be considered in patients with CURB65 scores $<2$ who require hospitalisation for reasons such as comorbidities (B II).

- Sputum should not be submitted on patients with CAP who are being treated as outpatients (A II).

- The use of the pneumococcal UAT is not routinely recommended for patients with CAP (B II). 
- The Legionella UAT should be considered, where available, for patients with severe CAP (B III).

- The use of rapid antigen tests for influenza is not recommended (B II).

- In patients with severe CAP during the influenza season (typically June to September) nasopharyngeal samples may be considered for detection of influenza (B II).

- The routine use of molecular tests to detect additional pathogens is not recommended (B II).

- Serology for 'atypical' pathogens should not be routinely performed (A II).

\section{Investigating for tuberculosis}

South Africa faces a huge burden of TB; this is driven mainly by high rates of HIV infection (over 50\% of incident TB cases are HIV-infected) and a large mining workforce $(76,77)$. The National Department of Health (NDOH) guidelines recommend annual TB screening for all individuals in South Africa; this is supported by the WHO policy of intensified case finding for TB control in high prevalence regions. TB is a cause of CAP and the clinical features are not reliable in distinguishing TB from other aetiologies. However, TB should be suspected in patients presenting with CAP who are HIV-infected or who have diabetes mellitus $(78,79)$, in any patient admitted to ICU (80), and in those with subacute illness or those not responding to empiric antimicrobial therapy.

Traditional diagnostic tests for TB have major drawbacks; automated liquid culture systems are the gold standard for the diagnosis of TB but are expensive and require prolonged incubation while sputum smear microscopy has a much lower diagnostic yield (81), particularly in HIV-infected patients, and does not provide drug susceptibility data. In response to these limitations, the NDOH recently rolled out GeneXpert MTB/RIF ${ }^{\oplus}$ (Xpert) (Cepheid, Sunnyvale, CA, USA) as a replacement for smear microscopy to diagnose pulmonary TB in South Africa. The assay has overall pooled sensitivities of $88 \%$ to detect culture-positive TB cases, and $67 \%$ after a negative smear microscopy result (82). A major advantage of the test is that it is able to rapidly detect patients with rifampicin resistance. Sputum induction provides an adequate sample and a bacteriological diagnosis more frequently than instruction by a health-care worker but is costlier and does not result in a higher proportion of same-day diagnoses (83). Other WHO endorsed and clinically validated rapid molecular TB tests are also in use in South Africa. Certain genotypic assays, such as line probe assays, may have diagnostic accuracy that is comparable to Xpert MTB/RIF and have the added advantage of detecting both isoniazid and rifampicin resistance as well as susceptibility to second line TB drugs (84-86).

There is a growing evidence base for urine-based TB lipoarabinomannan (LAM) testing in HIV-infected patients, and a rapid point of care lateral flow assay is now available. The test performs particularly well for HIV-infected patients with CD4 counts $<100$ cells $/ \mu \mathrm{L}$, and studies have shown a sensitivity of $\sim 40 \%$ for inpatients with confirmed TB $(87,88)$, with an ability to detect the sickest patients with advanced immunosuppression $(89,90)$. Appropriate training on LAM test performance and systems for quality control should be implemented in facilities where the test is in use.

\section{Recommendations}

- In the following high risk patient groups presenting with CAP there should be a low threshold for investigation for pulmonary TB: HIV-infected, diabetics, admission to ICU, subacute illness or those not responding to empiric antimicrobial therapy (A II).

- A GeneXpert MTB/RIFTM (Cepheid, Sunnyvale, USA) assay performed on a single expectorated or induced sputum specimen is the preferred first line diagnostic test for pulmonary TB. Alternatively, WHO endorsed rapid molecular tests, such as line probe assays, are recommended when they are more readily available (A II).

- TB culture should be performed in the following patients with a negative GeneXpert MTB/RIF: non-resolving pneumonia or an ongoing suspicion of TB (A III).

- When sputum is unavailable Determine ${ }^{T M}$ TB-LAM Ag (Alere, Waltham, MA, USA) testing should be performed in HIVinfected patients with CD4 counts $<100$ cells/ $\mu \mathrm{L}$ or stage 3 or 4 disease who present with CAP (A I).

\section{Investigating for pneumocystis pneumonia}

PCP typically presents as a subacute illness with constitutional symptoms and dry cough, and is characterised by bilateral infiltrates on CXR, normal chest auscultation and desaturation on pulse oximetry after minimal exertion (91). It may progress to respiratory failure and ARDS, and carries an overall case fatality of $15 \%$ (18).

The gold standard diagnostic test for PCP is immunofluorescent staining (IFA) of $P$. jirovecii organisms on bronchoalveolar lavage samples. This test requires an invasive procedure and is not widely available in South Africa, and therefore is rarely used to diagnose PCP. Immunohistochemical stains (IFA and silver stains) are most commonly requested on expectorated and induced sputum samples, but the sensitivity of these tests is poor $(\leq 60 \%)$ and they are inadequate to rule out the diagnosis of PCP (92). Sensitive PCR assays (93-97), including a commercial assay $(98,99)$, have been developed and evaluated on a variety of respiratory specimens. Unfortunately, HIV-associated PCP has not been well represented in these evaluation studies. These assays are not available for routine use in the public sector in South Africa and whilst having good sensitivity they have low specificity and are not routinely recommended.

Plasma [1,3]- $\beta-D$-glucan (beta-glucan) (100) and lactate dehydrogenase (LDH) $(101,102)$ have been used as supportive investigations but there is very limited evidence for their efficacy in South African settings. Therefore, the use of beta-glucan is only recommended as part of a clinical registry or trial. Because 
of these limitations, clinical assessment remains the most common method of diagnosis of PCP in SA, and should be based on the WHO case definition (Box 2) (103).

Box 2: WHO case definition for PCP in patients with HIV

Dyspnoea on exertion or non-productive cough of recent onset (within the past three months), tachypnoea and fever

And chest X-ray evidence of diffuse bilateral interstitial infiltrates

And no evidence of bacterial pneumonia: bilateral crackles on auscultation with or without reduced breath sounds

\section{Recommendations}

- The WHO clinical case definition should be used to clinically diagnose PCP (B III).

- Diagnostic testing of HIV-infected patients who fit the WHO case definition or in whom PCP is suspected on clinical grounds, depends on local availability of tests and may include an IFA, DFAT or PCR (B III).

- The preferred specimen for diagnostic tests for PCP is BAL although induced or expectorated sputum may be used when bronchoscopy is unavailable (B II).

- There is limited evidence to support for the use of beta-glucan to diagnose PCP in a South African setting. Its use is only recommended as part of a clinical registry or trial (A III).

\section{Antibiotic therapy}

\section{Initial antibiotics}

The choice of initial antibiotics for CAP in South Africa depends on the setting in which the patient is being treated, their age, use of antibiotics within the past 90 days and the presence of comorbidities (cardiovascular disease, chronic respiratory disease, chronic renal failure, diabetes mellitus and HIV infection) and drug intolerance. A recent systematic review found there were not enough trials to compare the effects of different antibiotics for pneumonia acquired and treated in the community (104) and guidance is therefore based on expert opinion (Tables 1,2). Figure 1 is an algorithm for the management of community-acquired pneumonia in adults in South Africa.
The reason that the presence of comorbidities was considered an important reason for modifying and broadening antibiotic treatment in the current guideline relates to a number of studies that have been undertaken documenting that they are associated with distinct aetiological patterns in patients with CAP, frequently associated with a broader spectrum of pathogens (105-108). In the study by Ruiz and colleagues (105), comorbid pulmonary disease, hepatic and nervous system illnesses as well as current smoking and alcohol abuse were associated with distinct patterns of aetiology for each of those conditions. In that study, respiratory comorbidities were defined as treatment for asthma or COPD, or presence of interstitial lung disorders, hepatic comorbidities were defined as pre-existing viral or toxic hepatopathy and nervous system illnesses were defined as symptomatic acute or chronic vascular or nonvascular encephalopathy with or without dementia. For example, in that study patients with pulmonary disorders were at a greater risk of infections with gram-negative enteric bacilli and Pseudomonas aeruginosa. In the study by El-Solh et al.(106), activities of daily living index, and pulmonary (defined as treatment for COPD or interstitial lung disease), endocrine (defined as diabetes mellitus) and CNS (defined as symptomatic acute or chronic vascular or nonvascular encephalopathy) comorbidities were associated with similar distinct aetiological patterns. The study by Cilloniz and co-workers (107) documented that pathogens such as $S$. aureus, Enterobacteriaceae, Pseudomonas aeruginosa, and $H$. Influenzae only occurred in CAP patients with one or more comorbidities, which included one or more of the following; chronic respiratory disease, diabetes mellitus, chronic cardiovascular disease, neurologic disease, chronic liver disease, and chronic renal disease, with these comorbidities not being further defined. In addition, multiple medical comorbid conditions have been documented to be a risk factor for penicillin-resistant and other drug-resistant pneumococcal infections (108).

Patients treated at home who are $<65$ years old, without antibiotic exposure in the past 90 days or comorbidities should receive oral high dose amoxicillin. Patients treated at home who are $\geq 65$ years old, have received antibiotics within the previous 90 days or who have comorbidities should receive oral amoxicillin-clavulanate or an oral second generation

Table 1: Empiric choice of antibiotics for CAP

\begin{tabular}{|l|l|l|l|l|}
\hline Setting & Route & $\begin{array}{l}<65 \text { years old, no } \\
\text { antibiotics within } 90 \text { days } \\
\text { and no comorbidities }\end{array}$ & $\begin{array}{l}\geq 65 \text { years, antibiotics with } 90 \text { days or } \\
\text { comorbidity* }\end{array}$ & Alternative \\
\hline Outpatient & PO & Amoxicillin & $\begin{array}{l}\text { Amoxicillin-clavulanate or a second } \\
\text { generation cephalosporin }\end{array}$ & Moxifloxacin or levofloxacin \\
\hline $\begin{array}{l}\text { Inpatients } \\
\text { (non-severe) }\end{array}$ & PO/IV & Ampicillin & $\begin{array}{l}\text { Amoxicillin-clavulanate or cefuroxime or } \\
\text { a third generation cephalosporin }\end{array}$ & Moxifloxacin or levofloxacin \\
\hline $\begin{array}{l}\text { Inpatients } \\
\text { (severe/ICU) }\end{array}$ & IV & $\begin{array}{l}\text { Amoxicillin-clavulanate } \\
\text { or cefuroxime or a third } \\
\text { generation cephalosporin } \\
\text { plus a macrolide/azalide }\end{array}$ & $\begin{array}{l}\text { Amoxicillin-clavulanate or cefuroxime or } \\
\text { a third generation cephalosporin plus a } \\
\text { macrolide/azalide }\end{array}$ & $\begin{array}{l}\text { Moxifloxacin or levofloxacin plus } \\
\text { amotillin-clavulanate or cefuroxime or }\end{array}$ \\
\hline
\end{tabular}

*, cardiovascular disease, chronic respiratory disease, chronic renal failure, diabetes mellitus, HIV infection. CAP, community-acquired pneumonia. 
cephalosporin. In both groups the alternative is an oral respiratory fluoroquinolone when there is severe beta-lactam allergy.

Patients admitted to hospital should be treated with intravenous ampicillin or penicillin in the first instance unless they are $\geq 65$ years, have recent antibiotic exposure or co-morbidities when amoxicillin-clavulanate, cefuroxime or a third generation cephalosporin (ceftriaxone or cefotaxime) should be used. An alternative is a respiratory fluoroquinolone which is equally effective give orally or intravenously (109).

There is emerging evidence in patients with more severe pneumonia of either pneumococcal or non-pneumococcal aetiology, and including critically ill cases, that combination antibiotic therapy, most commonly the addition of a macrolide agent to standard beta-lactam therapy, may be associated with a better outcome than monotherapy $(110,111)$. Recent systematic reviews and meta-analyses of critically ill patients with CAP, comparing macrolide-based therapies with other regimens clearly indicated that macrolide use was associated with a significant reduction in mortality compared with non-macrolide containing regimens, and the benefit became even more significant with the pooling of data from studies that provided adjusted risk estimates $(112,113)$. This mortality benefit from the use of macrolide-based combination antibiotic regimens versus other antibiotic regimens in critically ill patients was also supported by studies of survival among intubated patients with CAP (114) and among CAP patients with severe sepsis, the latter even when evaluating patients with CAP infections due to macrolide-resistant pathogens (e.g., macrolide-resistant pneumococci and gram-negative pathogens) (115). Patients with severe CAP should therefore be treated with a combination of amoxicillin-clavulanate, cefuroxime or a third generation (ceftriaxone or cefotaxime) cephalosporin plus a macrolide/ azalide antibiotic. An alternative regimen for severe CAP is a respiratory fluoroquinolone, which should be combined with another agent, most commonly a beta-lactam. In adults hospitalised with CAP, antibiotic therapy initiated within 4-8 hours of hospital arrival was associated with lower adjusted short-term mortality (113).

\section{Additional considerations}

In areas with epidemiologically high rates of penicillinresistant S. pneumoniae (PRSP) pharmacokinetically enhanced amoxicillin-clavulanate $(2,000 \mathrm{mg}$ amoxicillin-125 $\mathrm{mg}$ clavulanate 12-hourly) may be the preferred agent. Clinical studies with this formulation have demonstrated $97.1 \%$ bacteriological success against erythromycin-resistant (erythromycin MICs $>1 \mathrm{mg} / \mathrm{L}$ ) isolates and the formulation has also demonstrated high bacteriological efficacy against PRSP isolates non-susceptible to amoxicillin (amoxicillin MICs $>4 \mathrm{mg} / \mathrm{L}$ ), with $86.7 \%$ bacteriological success (116).

While significant macrolide resistance precludes the routine use of these agents as monotherapy for many patients with CAP, in areas of low macrolide resistance, and in young, otherwise healthy adults who have not recently received a course of antibiotics these agents may be used on their own, particularly in the setting of severe beta-lactam allergy. A macrolide/azalide/ tetracycline may also be used alone or in combination with another agent in cases suspected or known to be infected with so-called 'atypical' pathogens.

\section{Recommendations}

- Patients treated at home who are $<65$ years old, without antibiotic exposure in the past 90 days, or comorbidities, should receive oral high dose amoxicillin (A II).

- Patients treated at home who are $<65$ years old, without antibiotic exposure in the past 90 days, or comorbidities, in the setting of low macrolide resistance, could receive an oral macrolide/azalide in the presence of severe beta-lactam allergy (A II).

- Patients treated at home who are $\geq 65$ years old, have received antibiotics within the previous 90 days, or who have comorbidities, should receive oral amoxicillin-clavulanate or an oral second generation cephalosporin (A II).

- Patients whose admission to hospital is precipitated by advanced age, personal or family preference, inadequate home care or adverse social circumstances who have nonsevere pneumonia, can be treated with oral antibiotics as described above (A II).

- Patients requiring admission to hospital who are $<65$ years old, without antibiotic exposure in the past 90 days, or comorbidities, should receive intravenous ampicillin or penicillin (if IVI ampicillin not available) (A II).

- Patients requiring admission to hospital who are $\geq 65$ years old, have received antibiotics within the previous 90 days, or who have comorbidities, should receive intravenous amoxicillinclavulanate, cefuroxime or a third generation cephalosporin (ceftriaxone or cefotaxime) (A II).

- Patients with severe pneumonia should receive amoxicillinclavulanate or cefuroxime or a third generation cephalosporin (ceftriaxone or cefotaxime) plus a macrolide antibiotic (A II).

- Respiratory fluoroquinolones (moxifloxacin or levofloxacin) are an alternative therapy but because of their activity against tuberculosis these agents should not be used as first line in CAP. They may be used in patients with severe beta-lactam allergy or as an alternative to beta-lactam/macrolide therapy but should be reserved for use in patients who have no alternative treatment options (A II).

- Antibiotics should be administered early, preferably within the emergency unit, to patients with confirmed CAP (A II).

\section{Definitive therapy}

Although microbiological confirmation of the aetiology of CAP will only be obtained in the minority of cases, it is important that when a causative organism is identified, antibiotics are changed to the narrowest spectrum agent that effectively treats the organism. When the causative organism is resistant to initial therapy, it is necessary to use a broader spectrum agent. 
Table 2: Dose and route of administration for antibiotics prescribed for CAP

\begin{tabular}{|c|c|c|c|}
\hline Antibiotics & Route & Dose & Time of administration \\
\hline \multicolumn{4}{|l|}{ Penicillins } \\
\hline Benzylpenicillin (S. pneumoniae MIC $\leq 0.5$ mg/L) & IV & $2 \mathrm{MU}$ & 6 hourly \\
\hline Benzylpenicillin (S. pneumoniae MIC $\leq 1$ mg/L) & IV & $4 \mathrm{MU}$ & 6 hourly \\
\hline Benzylpenicillin (S. pneumoniae MIC $\leq 2 \mathrm{mg} / \mathrm{L}$ ) & IV & $4 \mathrm{MU}$ & 4 hourly \\
\hline Amoxicillin & PO & $1 \mathrm{~g}$ & 8 hourly \\
\hline \multirow[t]{3}{*}{ Amoxicillin-clavulanate } & $\mathrm{PO}$ & $1 \mathrm{~g}$ & 8 hourly \\
\hline & $\mathrm{PO}$ & $2 \mathrm{gSR}$ & 12 hourly \\
\hline & IVI & $1.2 \mathrm{~g}$ & 8 hourly \\
\hline Amoxicillin-clavulanate/plus amoxicillin & PO & $375 \mathrm{mg} /$ plus $500 \mathrm{mg}$ & 8 hourly/8 hourly \\
\hline Ampicillin & IVI & $1-2 g$ & 6 hourly \\
\hline \multicolumn{4}{|l|}{ Cephalosporins } \\
\hline Cefuroxime axetil & PO & $750 \mathrm{mg}$ & 8 hourly \\
\hline Cefuroxime & IVI & $1.5 \mathrm{~g}$ & 8 hourly \\
\hline Cefpodoxime & PO & $400 \mathrm{mg}$ & 12 hourly \\
\hline Ceftriaxone & IVI & $1-2 g$ & 24 hourly \\
\hline Cefotaxime & IVI & $1-2 g$ & 8 hourly \\
\hline Ceftaroline & IVI & $600 \mathrm{mg}$ & 12 hourly \\
\hline \multicolumn{4}{|l|}{ Fluoroquinolones } \\
\hline \multirow[t]{2}{*}{ Moxifloxacin } & PO & $400 \mathrm{mg}$ & Daily \\
\hline & IVI & $400 \mathrm{mg}$ & Daily \\
\hline \multirow[t]{2}{*}{ Levofloxacin } & PO & $750 \mathrm{mg} / 500 \mathrm{mg}$ & Daily/12 hourly \\
\hline & IVI & $750 \mathrm{mg} / 500 \mathrm{mg}$ & Daily/12 hourly \\
\hline \multicolumn{4}{|l|}{ Macrolides/azalides } \\
\hline \multirow[t]{2}{*}{ Erythromycin } & PO & $500 \mathrm{mg}$ & 6 hourly \\
\hline & IVI & $1 \mathrm{~g}$ & 6 hourly \\
\hline \multirow[t]{3}{*}{ Clarithromycin } & $\mathrm{PO}$ & $500 \mathrm{mg}$ & 12 hourly \\
\hline & PO & $1 \mathrm{gXL}$ & Daily \\
\hline & IVI & $500 \mathrm{mg}$ & 12 hourly \\
\hline \multirow[t]{2}{*}{ Azithromycin } & $\mathrm{PO}$ & $500 \mathrm{mg}$ & Daily \\
\hline & IVI & $500 \mathrm{mg}$ & Daily \\
\hline \multicolumn{4}{|l|}{ Tetracyclines } \\
\hline Doxycycline & PO & $200 \mathrm{mg} / 100 \mathrm{mg}$ & Stat $(200 \mathrm{mg}$ ) followed by $100 \mathrm{mg} 12$ hourly \\
\hline \multicolumn{4}{|l|}{ Carbapenems } \\
\hline Ertapenem & IVI/IM & $1 \mathrm{~g}$ & Daily \\
\hline
\end{tabular}

CAP, community-acquired pneumonia. 


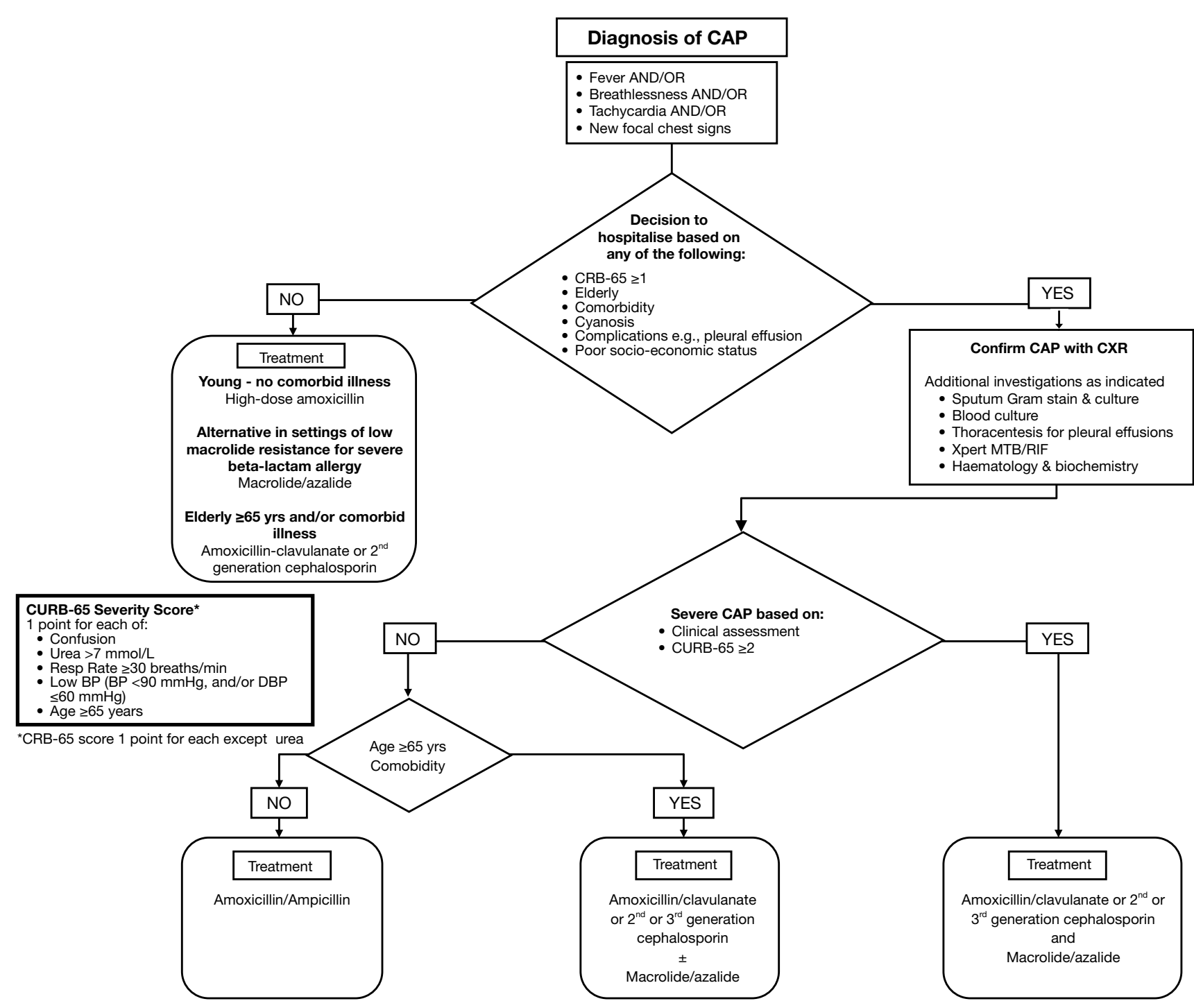

Figure 1: Algorithm for the management of community-acquired pneumonia in adults in South Africa. This figure should be read in conjunction with the text. Adapted with permission from the South African Medical Journal (S Afr Med J 2007;97:1295-306).

Although standard agents may be used in patients with highlevel resistance, two agents that should not be used as empiric therapy may be used.

Ceftaroline fosamil is a new, broad-spectrum cephalosporin prodrug that exhibits bactericidal activity against gram-positive pathogens, including methicillin-resistant Staphylococcus aureus (MRSA) and S. pneumoniae, as well as gram-negative pathogens. Integrated analysis of two registrational studies (Ceftaroline Community Acquired Pneumonia Trial versus Ceftriaxone in Hospitalized Patients) (FOCUS 1 and FOCUS 2), that compared ceftaroline with ceftriaxone in the treatment of adult patients requiring hospitalisation for CAP, demonstrated clinical cure rates for the ceftaroline group that were numerically higher than those for the ceftriaxone group and that this agent was well tolerated, with a safety profile similar to that of ceftriaxone (117). Ceftaroline should be reserved for use in patients with microbiologically confirmed CAP due to penicillinresistant S. pneumoniae (penicillin MIC $\geq 8 \mathrm{mg} / \mathrm{L}$ ) or MRSA.

Ertapenem, a group 1 carbapenem (limited activity against non-fermentative gram-negative bacilli) has excellent in vitro activity (i.e., $\geq 90 \%$ of isolates have an ertapenem MIC less than or equal to the susceptibility breakpoint) against bacteria that, in general, are associated with CAP, such as S. pneumoniae, methicillin-susceptible Staphylococcus aureus (MSSA) and many Haemophilus spp., Enterobacteriaceaeincluding extended-spectrum $\beta$-lactamase (ESBL) producing strains and anaerobes (118). The efficacy and safety of ertapenem, for the treatment of CAP requiring parenteral therapy, were compared with those of ceftriaxone in two registration trials and demonstrated that ertapenem, $1 \mathrm{~g}$ once a day, was highly effective therapy for CAP in hospitalised adults with moderateto-severe disease (118). Ertapenem should be reserved for use in patients with microbiologically-confirmed CAP due to resistant Enterobacteriaceae, such as ESBL-producing pathogens.

\section{Recommendations}

- When a causative organism is identified by microbiological testing, antibiotics should be changed to the narrowest spectrum agent that effectively treats the organism (A II). 
- Ceftaroline is recommended as directed therapy based on the results of microbiological testing in cases of penicillinresistant S. pneumoniae (penicillin MIC $\geq 8 \mathrm{mg} / \mathrm{L}$ ) or MRSA (A I).

- Ertapenem is recommended as directed therapy based on the results of microbiological testing in cases of resistant Enterobacteriaceae such as ESBL-producing pathogens (A I).

\section{When to add empiric therapy for PCP and TB}

The WHO case definition of PCP in patients with HIV is shown in Box 2. Patients with HIV infection who fulfill these criteria or with a positive specific test for PCP should be initiated on therapy

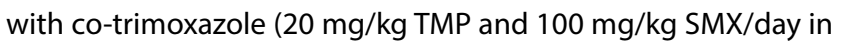
divided doses) orally or intravenously and prednisone $40 \mathrm{mg}$ twice daily for 5 days followed by 40 mg daily for 5 days and then $20 \mathrm{mg}$ daily for 11 days.

\section{Recommendations}

- Empiric therapy for PCP should be added when patients fulfill the WHO case definition and it should not be withheld on the basis of negative immunohistochemical staining on sputum specimens (A II).

- Empiric therapy for TB prior to initial testing is rarely required unless there is a miliary pattern on CXR or the patient is severely ill and TB is suspected (A III).

\section{When to add empiric therapy for influenza}

The influenza season in South Africa typically starts in early June and runs until around September. Up to date information is available from the National Institute for Communicable Diseases website (www.NICD.ac.za). During this period influenza should be considered in any patient with severe pneumonia, particularly if there is a history of a preceding upper respiratory tract infection and/or diffuse bilateral infiltrates on CXR (119). If influenza is suspected on these grounds patients should be initiated on oseltamivir (75 mg twice daily) $(120,121)$ and a nasopharyngeal aspirate tested for influenza by polymerase chain reaction. Specific risk factors for severe influenza are pregnancy, immune compromise (including diabetes mellitus and HIV infection), obesity and chronic lung, cardiac, neurological disease and age $>65$ years $(122,123)$.

\section{Recommendations}

- During the influenza season oseltamivir should be provided for any patient with severe pneumonia and can be stopped if PCR testing of nasopharyngeal aspirate is negative (A II).

- During the influenza season oseltamivir should be provided for any patient with moderate CAP who is suspected of having influenza if they have a specific risk factor for severe disease and can be stopped if PCR testing of nasopharyngeal aspirate is negative (B II).

\section{Adjunctive therapies}

Since the mortality of patients with CAP, particularly those who need hospitalisation, and especially those in the intensive care unit remains high, even in the presence of effective antibiotic therapy, studies have been ongoing to find effective adjunctive therapies that could be used together with antibiotics to improve the outcome (124-126). Multiple agents have been recommended or tested but results have largely been very disappointing.

\section{Statins}

While prior statin use has been shown in systematic reviews and meta-analyses to be associated with a decreased risk and/ or mortality of CAP (127-130), there is not enough evidence from randomised controlled trials to recommend their routine use to either prevent CAP or to improve its mortality. The only randomised, double-blind, placebo-controlled, intervention study investigating the impact of statin use on admission of patients with CAP to hospital was not associated with a reduction in cytokine levels, nor was it associated with a reduction in time to clinical stability among the patients (131).

\section{Corticosteroids}

Several recent studies and meta-analyses, measuring different end-points, have shown definite benefits of adjunctive corticosteroids in severe hospitalised patients with CAP (132-139). The data generated from these studies and systematic reviews have recently been extensively reviewed (140). One of the most comprehensive meta-analyses was that of Siemieniuk and colleagues (138), who in addition to undertaking an extensive extraction of the literature, analysed the data for all possible benefits and potential harms, using instruments to assess risk of bias in the individual studies, as well as publication bias, and the GRADE system to evaluate the quality/certainty of the evidence. The final assessment of findings was that corticosteroid use was associated with a lower mortality (significant only in the severe CAP group), reduction in need for mechanical ventilation, the occurrence of ARDS, time to clinical stability and length of hospital stay with the evidence being of moderate or higher quality (138). There are still unanswered questions regarding corticosteroid use, including which patients with CAP are most likely to benefit, which corticosteroids to use, at what dose and for how long. However, the data from the various studies and meta-analyses suggest that those with severe CAP requiring ICU admission, those with the highest inflammatory indices (such as a CRP level above $150 \mathrm{mg} / \mathrm{L}$ ) and those with shock requiring vasopressor support are most likely to benefit (140). The recommended regimen is methylprednisone $0.5 \mathrm{mg} / \mathrm{kg} / 12 \mathrm{~h}$ or equivalent for 5 days (135). While patients with diabetes (141) and those receiving long-term steroids have been included in $\mathrm{RCTs}$, there are limited data in patients with HIV infection.

\section{Recommendations}

- There is not enough evidence to recommend the routine use of statins in CAP (A I). 
- The addition of a macrolide to standard beta-lactam therapy is associated with a better outcome in patients with severe CAP requiring ICU admission and while this may relate to the antimicrobial activity of macrolides, it may also be due to their anti-inflammatory, immunomodulatory effects (A II).

- Use of systemic corticosteroids (e.g., methylprednisone $0.5 \mathrm{mg} / \mathrm{kg} / 12 \mathrm{~h}$ or equivalent) should be considered in patients with severe CAP requiring ICU admission unless influenza or tuberculosis is likely, or there is a history of gastro-intestinal bleeding within the previous 3 months (A I).

\section{Severely ill patients with CAP}

Mortality is in the region of $12 \%$ for hospitalised CAP but $>30 \%$ among those admitted to the ICU (142).

Obvious reasons for referral are the need for mechanical ventilation and the presence of septic shock. Otherwise patients with a CURB-65 of $\geq 3$ should be evaluated for ICU admission. Clinical judgment, however, is important as elderly or immunocompromised patients may warrant ICU admission even with lower scores (143).

Organisms that cause severe CAP are similar to those that cause less severe disease; S. pneumoniae, Legionella spp., $S$. aureus and $K$. pneumoniae and viruses such as influenza (especially in unvaccinated patients, asthmatics, the obese, in immunocompromised cases and in pregnancy). However, other organisms are important to consider especially in the right clinical or geographical context: influenza H5N1 and H7N3, SARS and MERSCoV, Hantavirus, P. jirovecii, enteric gram-negative bacilli (elderly, aspiration), MSSA or MRSA (influenza, travel, corticosteroids, diabetes), and M. tuberculosis.

Potentially useful interventions include those that are relevant to any patient with severe sepsis and mechanical ventilation should be utilised to restore adequate oxygenation without causing lung injury $(144,145)$.

Antibiotics should be administered as soon as the diagnosis is made, preferably in the emergency room. These are similar to those described above except that intravenous macrolides should be administered to all severe pneumonias, particularly in the presence of septic shock, because of their immunomodulatory effect and because coincidentally they would cover L. pneumophila whether or not it is initially suspected. It does not appear, however, that empiric antibiotic coverage of other atypical pathogens such as $M$. pneumoniaeand C. pneumoniae improves survival or clinical efficacy in hospitalised patients (146). In an influenza season oseltamivir should be initiated in any patient with severe pneumonia in whom influenza is suspected and stopped once it has been excluded by PCR. In patients with influenza pneumonia, the earlier that the neuraminidase inhibitor is started the better the outcome (147).

Severe pneumonia is itself a form of primary ARDS. However, spreading infiltrates may represent secondary ARDS due to capillary leak and not antibiotic failure. Where diagnostic uncertainty exists, particularly in immunocompromised patients with bilateral infiltrates and where pneumocystis PCR is unavailable, the use of biomarkers, including $C R P, P C T$, proBNP and BDG \{plasma [1,3]- $\beta-D-$ glucan (beta-glucan)\} is extremely helpful in elucidating the cause and decreasing unnecessary antibiotic prescription. Biomarkers may also help to identify antibiotic failure. A declining CRP generally indicates that the antibiotics are appropriate despite worsening radiological features (148).

\section{IVPOS and duration of antibiotics}

Early IVPOS of antibiotics is a central pillar of antibiotic stewardship as it reduces costs and intravenous cannula infections and encourages reduced length of hospital stay (149). Several studies have shown that it is safe to switch patients with CAP from intravenous to oral therapy when they become clinically stable $(150,151)$. Definitions of clinical stability vary and a typical definition is given in Box 3 (151).

The precise duration of antibiotic therapy for the management of microbiologically documented and non-documented CAP is not informed by robust evidence. The duration of therapy should be determined based on the clinical response of the patient and the causative agent. When fever defervesces rapidly and there is clinical improvement it is safe to stop beta-lactam antibiotics after 5-7 days (6).

In patients who show a slow clinical improvement or who have a confirmed aetiological agent such as Pseudomonas aeruginosa, S. aureus or gram-negative enteric organisms, it may be necessary to continue antibiotics for longer.

\section{Recommendations}

- Patients can switch from intravenous to oral antibiotics when they are haemodynamically stable, have respiratory rate $<25 /$ min, temperature $<37.8^{\circ} \mathrm{C}$ and are able to take oral medication (A I).

- For community managed, and for most patients admitted to hospital with low or moderate severity and uncomplicated

Box 3: Features of clinical stability such that patients with CAP can be safely switched from intravenous to oral antibiotics

\begin{tabular}{l} 
Haemodynamically stable \\
Heart rate $<100 / \mathrm{min}$ \\
No need for intravenous fluid administration \\
Respiratory stable \\
Respiratory rate $<25 / \mathrm{min}$ \\
Oxygen saturation $>92 \%$ without $\mathrm{O}_{2}$ \\
\hline Free of fever-temperature $<37 . \mathbf{8}^{\circ} \mathbf{C}$ \\
\hline Free of delirium \\
Able to take oral medication \\
The patient is able to swallow \\
No vomiting \\
No diarrhoea
\end{tabular}

CAP, community-acquired pneumonia. 
pneumonia, 5-7 days of appropriate antibiotics is recommended (A II).

- Treatment duration may be extended beyond 5-7 days for specific clinical scenarios such as Staphylococcus aureus bacteraemia (A II).

- Patients with confirmed Legionella pneumonia should be treated with azithromycin for 7 days (A II).

\section{Acute complications of CAP}

Most cases of pneumonia resolve completely with appropriate antibiotic treatment and supportive care. However, a number of important complications of CAP may occur that require specific management. These complications should be considered whenever a patient fails to respond adequately to therapy, although some patients present later with general ill health and ongoing constitutional symptoms. The diagnosis of complications of CAP is frequently delayed and clinicians should have a low threshold for investigations.

\section{Complicated para-pneumonic effusion and empyema}

Para-pneumonic effusions occur in at least $40 \%$ of bacterial CAP, and are usually small (152). They are characterised by exudative chemistries and an influx of neutrophils into the pleural space. Most effusions are uncomplicated and resolve with treatment of the pneumonia. However, if bacteria invade the pleural space, a complicated para-pneumonic effusion or empyema results. Anaerobic utilisation of glucose by the neutrophils results in pleural fluid acidosis, and lysis of neutrophils increases the LDH concentration in the pleural fluid to values often more than 1,000 IU/L. Empyema develops when there is evidence of bacterial infection of the pleural liquid, as indicated by the presence of pus in the pleural space and/or the presence of bacterial organisms on Gram stain. A positive culture is not required for diagnosis, since there are several reasons why bacteria may not be cultured from an empyema, including the presence of anaerobic organisms in the pleural space that may be difficult to culture. The characteristic putrid odour of the pleural fluid is classically considered diagnostic of anaerobic infection.

In a patient with non-resolution of CAP, the demonstration of any significant amount of pleural fluid on CXR should prompt diagnostic pleurocentesis. The fluid is considered an exudate if any of the following are found and a transudate if all are absent (Light's criteria) (153):

- Ratio of pleural fluid to serum protein $>0.5$.

- Ratio of pleural fluid to serum LDH >0.6.

- Pleural fluid LDH > two thirds of the upper limits of normal serum value.

In a more recent systematic review, pleural fluid cholesterol greater than $55 \mathrm{mg} / \mathrm{dL}$ and pleural LDH greater than $200 \mathrm{U} / \mathrm{L}$ each had better positive and negative likelihood ratios for distinguishing exudates from transudates than did Light's criteria (154).
A low pleural fluid $\mathrm{pH}$ level is more predictive of complicated effusions (that require drainage) than is a low pleural fluid glucose level. In such cases, a pleural fluid $\mathrm{pH}$ of $<7.2$ indicates the need for urgent drainage of the effusion, if $\mathrm{pH}$ is $7.2-7.3$ it is reasonable to observe the patient while a pleural fluid $\mathrm{pH}$ of $>7.3$ suggests that the effusion may be managed with systemic antibiotics alone $(155,156)$. Fluid drainage by mean of an intercostal drain is necessary in all cases of complicated para-pneumonic effusion or empyema. Current international guidelines strongly recommend the routine use of ultrasonography for all pleural fluid drainage procedures.

\section{Recommendations}

- Repeat CXR should be performed for any patient failing to respond to the first few days of empiric therapy or who deteriorates after an initial improvement (A II).

- If follow-up CXR demonstrates effusion or lung abscess, further imaging with $\mathrm{CT}$ or thoracic ultrasonography should be considered (B II).

- Any significant amount of pleural fluid should prompt diagnostic pleurocentesis to exclude empyema (A II).

- Fluid drainage by mean of an intercostal drain is necessary in all cases of complicated para-pneumonic effusion or empyema (A II).

\section{Lung abscess}

Lung abscess is defined as necrosis of the pulmonary parenchyma with cavitation. Acute lung abscess, associated with a short duration of symptoms and a rapidly-evolving chest radiograph, is a rare complication of CAP. Risk factors include immunosuppression, inappropriate antibiotic selection, and infection with S. aureus and K. pneumoniae. Acute lung abscess should be differentiated from a chronic abscess (presenting with indolent symptoms that evolve over a period of weeks or months) seen most commonly in the debilitated or alcoholic patient, and following aspiration in a patient with a reduced level of consciousness or impaired swallowing mechanisms. Infection with anaerobic bacteria, S. aureus, gram-negative enteric bacilli or S. anginosus group (previously known as S. milleri) in the presence of poor dental hygiene should be considered in that instance.

A lung abscess is typically diagnosed when a chest radiograph reveals a pulmonary infiltrate with a cavity; an air-fluid level is frequently present. Better anatomic definition can be achieved with a CT scan, which can distinguish a cavitating lung lesion from a pleural collection, also a complication of CAP. It may also demonstrate previously unrecognised underlying conditions such as an aspirated foreign body, a pulmonary neoplasm, or bronchial stenosis. Most patients respond to appropriate antibiotics guided by the microbiology of the precipitating episode of CAP. If patients fail to respond to antibiotics it may be necessary to insert a pigtail drain. Antimicrobial therapy with amoxicillin-clavulanate is appropriate if a chronic abscess is suspected. In this instance, sputum culture is unreliable as it is contaminated by oral flora. A prolonged course of antibiotics 
(up to 6 weeks) may be required, although there is no evidence on the optimum duration of antimicrobial therapy. Monitoring response with serial CRP measurements may be useful in guiding the length of therapy. Percutaneous drainage of the abscess (guided by either ultrasound or CT) can be performed diagnostically and therapeutically in non-responders, and surgery is rarely required (less than $5 \%$ of cases in most series).

\section{Recommendations}

- Patients diagnosed with lung abscess as a complication of CAP should receive a prolonged course of antibiotics, usually 4-6 weeks, along with physiotherapy to effect postural drainage (B II).

\section{Cardiovascular events}

There is an emerging awareness of the possible occurrence of cardiovascular events in patients with CAP, with one of the earliest studies of cardiac changes in CAP having been undertaken in South Africa (157). With regard to the cardiac events these may include acute myocardial infarction (AMI), new or worsening cardiac failure and acute or worsening arrhythmia, occurring either alone or in combination, and have been documented in all-cause CAP, as well as in pneumococcal CAP specifically (158163). While these cardiovascular events are more common in the elderly and in those patients with underlying cardiac and other risk factors, there is also evidence that these events may also occur in younger patients without a history of clinical cardiac disease or obvious additional risk factors (162). With regard to the pathogenesis, it is being increasingly recognised that platelet activation may play a central role in CAP-associated AMI, raising the possibility that anti-platelet agents, such as aspirin, among many other agents, may be beneficial in preventing these events, as has been documented in at least one study in the elderly (164-166). Furthermore, the pathogenesis of these cardiac events in pneumococcal CAP is increasingly being understood $(167,168)$. It is recommended that any patients with CAP who have well-recognised risk factors for a cardiovascular event, or patients who are complicated by clinical failure, should be investigated for the possibility of a cardiovascular event. Importantly, such events, when they occur in patients with CAP, are associated with a poorer prognosis acutely, as well as a poorer prognosis and associated increased risk of cardiovascular events on long-term follow-up (169-171). This topic has been reviewed in more detail elsewhere (172).

\section{Recommendations}

- Patients with CAP with well-recognised risk factors for a cardiovascular event or cases that fail to show adequate clinical recovery should be investigated for the possibility of a cardiovascular event (A III).

\section{Long-term sequelae of lung damage}

CAP occasionally leads to bronchiectasis, particularly if recurrent or associated with an underlying anatomic or immune defect. Bronchiectasis is defined as abnormal dilation and distortion of the bronchial tree, and is characterised by chronic sputum production with persistent airflow limitation. Significant infection, particularly in childhood, can cause structural damage that impairs mucociliary clearance and facilitates chronic bacterial infection. Over time, persistent infection may then result in bronchiectasis. Aetiological agents that have been implicated in the original precipitating bronchial wall injury include Mycoplasma pneumoniae, Bordetella pertussis, adenovirus and measles. Tuberculosis remains an important cause of bronchiectasis in patients from high-burden communities and in those with HIV infection.

\section{Aspiration pneumonia}

\section{Definitions}

The term 'aspiration' refers to the abnormal entry of a large inoculum of exogenous substances or endogenous secretions into the lower airways. This may result in lung inflammation with associated clinical consequences or resolve spontaneously without any therapy. The clinical presentation depends on the type and volume of aspirated material, the frequency of aspiration and the host immune response. The major syndromes related to aspiration include (173) airway obstruction from inhalation of particulate matter (174), aspiration pneumonitis, a chemical injury caused by aspiration of sterile liquid gastric contents (or other noxious fluids), and (175) aspiration pneumonia, an infectious process caused by the inhalation of oropharyngeal secretions colonised by microorganisms (173-176). While most cases of CAP are caused by microaspiration of relatively virulent bacteria residing in the upper respiratory tract (177), aspiration pneumonia refers to a syndrome of bacterial pneumonia in susceptible patients with defective lower airway clearance mechanisms who aspirate a large inoculum of normally nonvirulent pathogens $(178,179)$.

Although some features of aspiration pneumonitis and aspiration pneumonia overlap, they represent distinct clinical entities in terms of pathophysiological mechanisms, clinical manifestations and treatment. So-called 'bland' aspiration, as occurs after haematemesis or aspiration of enteral feeds, may lead to CXR infiltrates but does not result in an inflammatory response in the lung and usually resolves spontaneously without antimicrobial therapy $(178,180,181)$. There is often a failure by clinicians to distinguish aspiration pneumonitis or bland aspiration from aspiration pneumonia and a tendency to consider all aspiration syndromes to be infectious, resulting in overuse of antimicrobials (173).

\section{Epidemiology and risk factors}

Accurate estimation of the prevalence of community-acquired aspiration pneumonia (CAAP) is limited by the lack of a standardised case definition, and because most studies do not distinguish aspiration pneumonitis from pneumonia (179). Observational studies from developed countries have found that up to $15 \%$ of CAP episodes may result from aspiration $(47,182-186)$. Stroke patients who aspirate have a 7-fold higher risk of developing pneumonia (187), which complicates $10 \%$ of 
acute strokes and is associated with a significantly increased risk of death (188). Patients with CAAP are more likely to be admitted to intensive care units $(189,190)$ and require mechanical ventilation than those with non-aspiration CAP, and have a significantly increased in-hospital mortality and length of stay $(182,191)$.

The primary predisposing mechanisms for aspiration include dysphagia and altered mental status (192), resulting in compromised glottic closure and cough reflexes $(175,176)$. Dysphagia is regarded as the most important risk factor for aspiration pneumonia; it is most commonly due to neurological and oesophageal disorders, but also complicates COPD (193) and the use of antipsychotic medications (194). Alcohol abuse and seizures are strongly associated with anaerobic aspiration pneumonia because of reduced levels of consciousness, poor oral hygiene, immune dysfunction and delayed presentations (179). Conditions such as dental caries, periodontal disease and gingivitis increase the risk of oropharyngeal colonisation with pathogenic organisms and a higher overall bacterial load, and are associated with a higher risk of aspiration pneumonia $(173,176,195)$. The elderly therefore represent a higher risk group (186) because of the more frequent neglect of oral hygiene (196) and higher rates of neurological disease (173). Nasogastric- or gastrostomy-tube feeding are independent risk factors for aspiration (197).

\section{Microbiology}

A dominant role for anaerobic organisms in aspiration pneumonia was suggested by early studies using animal models and invasive diagnostic procedures such as transthoracic and transtracheal needle aspiration (198). The most common isolates included Bacteroides melaninogenicus and other Bacteroides species, Fusobacterium nucleatum, and Peptostreptococcus spp. $(175,199,200)$, many of which produce beta-lactamases. The relevance of these early studies assessing the bacteriology of aspiration pneumonia has been questioned, with concerns about the sterility of sampling techniques employed (201) and the inclusion of patients with established complications such as lung abscess and empyema (173). More recent studies using protected specimen brushes to sample the lower respiratory tract of patients with severe aspiration pneumonia isolated bacterial pathogens in a minority of cases. In these studies S. pneumoniae, S. aureus, H. influenzae, and Enterobacteriaceae predominated, and no pathogenic anaerobic organisms were isolated $(202,203)$. In a group of institutionalised elders with severe aspiration pneumonia who underwent bronchoscopic sampling, gram-negative enteric bacilli were the predominant organisms isolated (49\%), followed by anaerobic bacteria (16\%), and S. aureus (12\%) (204). This shift in microbiological profiles may reflect a true decline in anaerobic infection due to improved social conditions and access to health care, but the overlap with organisms found in health-care associated pneumonia suggests an increased occurrence of aspiration in these settings.

\section{Clinical features and diagnosis}

The early clinical features of aspiration pneumonia are difficult to distinguish from other causes of CAP, particularly because the aspiration event is usually not witnessed (175). The diagnosis is usually made in patients presenting with a pneumonic process with a predisposition for aspiration (i.e., difficulty swallowing or a reduced level of consciousness), plus involvement of dependent pulmonary segments (posterior segments of the upper lobes and the apical segments of the lower lobes when aspiration occurs in the recumbent position, or the basal segments of the lower lobes in an upright position), particularly in the right lower lobe $(202,203)$. There are no clinical or biochemical findings that reliably distinguish anaerobic aspiration pneumonia from CAP $(176,205)$, but the following may suggest an anaerobic cause of pneumonia (173-176,206):

- The production of foul-smelling sputum, suggesting infection with anaerobic organisms.

- The development of lung abscesses, necrotising pneumonia or empyema; the onset of these complications may be indolent, occurring around 2 weeks after the aspiration event.

In contrast, aspiration pneumonitis is a hyperacute illness that usually occurs after a witnessed aspiration event with rapidonset dyspnoea (within 2 hours of aspiration), bronchospasm, bilateral patchy CXR infiltrates (including non-dependent areas), and frothy sputum. Hypoxia is a prominent feature, and patients may progress to develop acute respiratory distress syndrome. This may be accompanied by a systemic inflammatory response with fever, leukocytosis and tachycardia, despite the absence of infection $(175,207)$. Based on animal models, more than $120 \mathrm{~mL}$ of gastric contents need to be aspirated to induce chemical pneumonitis in an average-sized adult (179), and so a witnessed large aspiration event supports this diagnosis.

\section{Antimicrobial therapy}

The frequent finding of anaerobic infections in CAAP in microbiological studies performed in the 1970s led to a change in recommendations for empiric antibiotic therapy for aspiration pneumonia, with a shift away from penicillin to the use of agents with specific anaerobic coverage such as clindamycin, metronidazole and beta-lactam/beta-lactamase inhibitor combinations. Small RCTs conducted in the 1980s and early 1990s comparing penicillin to clindamycin for patients with lung abscess and confirmed anaerobic pneumonia showed much better cure rates with the use of clindamycin $(208,209)$.

\section{Recommendations}

- Acute aspiration events, particularly in the absence of systemic inflammation or impaired respiratory function, do not require antimicrobial therapy, even if associated with a new CXR infiltrate (A III).

- Antimicrobials should be considered for patients with aspiration pneumonitis and persistent or progressive signs and symptoms 48 hours after aspirating (B III). 
- Aspiration pneumonia may be a more indolent process, usually occurring late after the aspiration event, and may be associated with suppurative complications. The diagnosis implies bacterial infection of the lung, and is therefore an indication for antimicrobial therapy (A II).

- Recommended empiric antibiotic therapy is amoxicillinclavulanate; a cephalosporin plus clindamycin or metronidazole may be an acceptable alternative (B II).

\section{Vaccination to prevent CAP}

Implementation of the South African National Guidelines for vaccination against influenza and pneumococcal infections may both assist in preventing CAP (see www.pulmonology.co.za).

Two pneumococcal vaccines are registered for use in adults in South Africa, the 23-valent polysaccharide vaccine (PPV23), and the 13-valent pneumococcal conjugate vaccine (PCV13).

The indications for PPV23 reported in the South African pneumococcal vaccination guideline are similar to that reported in the most recent Advisory Committee on Immunization Practices (ACIP) recommendations (210). In brief, PPV23 is recommended for use in adults aged 19-64 years with underlying comorbid conditions, who smoke cigarettes, have functional or anatomical asplenia and in those with immunocompromising conditions such as HIV infection, haematological malignancies and transplant patients. It is also recommended for use in persons 65 years of age or older.

In 2014, the PCV13 was registered for use in adults in South Africa, as in other parts of the world, based on initial immunogenicity studies. The initial registered indication in South Africa was the use of a single dose of PCV13 in adults $\geq 50$ years, as had been registered elsewhere (211). However, at the end of 2015, PCV13 received registration in South Africa for use in adults aged 18 years and older as a single dose (212). Specific groups at high risk for pneumococcal infection were mentioned in the registration including cases with sickle cell disease and HIV infection that were recommended to receive at least one dose of PCV13 whether or not they had received one or more doses of PPV23 previously. A specific regimen was recommended for patients with haematopoietic stem cell transplants.

Elsewhere in the world, such as in the USA, PCV13 has also been registered for use in adults $\geq 19$ years with underlying comorbid and immunocompromising conditions (213), and in adults $\geq 65$ years (214). In those with high-risk factors for pneumococcal disease (e.g., CSF leak, cochlear transplant and functional or anatomical asplenia) and those with immunocompromising conditions, as well as those aged $\geq 65$ years of age, the recommendation is for the use of PCV13 in sequence with PPV23. The recommendation is that PCV13 should always preferably be given first. In individuals who have not previously had PPV23 vaccination the PCV13 vaccine should be given first, followed a minimum of 2 months later with the PPV23 vaccine in the case of adults $\geq 19$ years of age with the high-risk underlying comorbid and immunocompromising conditions, and 12 months later in individuals $\geq 65$ years. However, in any of these cases, if the individual has already had a vaccination with PPV23, the PCV13 vaccination should be given a minimum of 1 year after the PPV23 vaccination.

Two recent clinical studies attest to the clinical efficacy of the PCVs in adults in different settings. The first study was that of the older 7-valent pneumococcal conjugate vaccine (PCV7) in predominantly HIV-infected adults and adolescents (aged $>15$ years of age) in Malawi, who had recently recovered from an episode of invasive pneumococcal disease (215). This was a randomised, double-blind, placebo controlled trial and in the active arm of the study, two doses of PCV7 were given to the patients 4 weeks apart. The efficacy of the vaccine for the primary endpoint, which was prevention of a further episode of vaccine serotype (or serotype $6 \mathrm{~A}$ ) pneumococcal infection, was $74 \%$ (95\% Cl, 30-90\%). The second was a study in The Netherlands among adults $>65$ years which evaluated the efficacy of PCV13 in preventing first episode of vaccine type strains of communityacquired pneumococcal pneumonia (primary endpoint), nonbacteremic and noninvasive pneumococcal CAP and invasive pneumococcal disease (secondary endpoints) (216). Patients in the active arm were given one dose of PCV13. Vaccine efficacy for the primary endpoint was $45.6 \%(95.2 \% \mathrm{Cl}, 21.8 \%$ to $62.5 \%)$, and for the secondary endpoints $45.0 \%(95.2 \% \mathrm{Cl}, 14.2 \%$ to $65.3 \%$ ) and $75 \%(95.2 \% \mathrm{Cl}, 41.4 \%$ to $90.8 \%)$, respectively. Efficacy persisted throughout the duration of the study of almost 4 years.

The trivalent inactivated influenza vaccine is the only licensed influenza vaccine available in South Africa (121). Because of the changing nature of influenza viruses, each year the strains included in the vaccine for the following influenza season are updated. For this reason, as well as limited duration of effectiveness, influenza vaccination is recommended to be repeated each year. Influenza vaccine usually becomes available in South Africa from March and should be given sufficiently early to provide protection for the winter season. Influenza vaccine effectiveness depends on characteristics of those being vaccinated (age and health), whether there is a good match between the circulating viruses and the viruses contained in the vaccine, and on influenza types and subtypes. In general, influenza vaccines work best among children $\geq 2$ years and healthy adults. Older people ( $\geq 65$ years), children $<2$ years and severely immunocompromised individuals often have poorer immune responses to trivalent inactivated influenza vaccine (IIV) compared with healthy adults. However, even for these people the influenza vaccine still provides some protection. Other products, e.g., high-dose influenza vaccine and adjuvant vaccines, have been shown to be more effective in certain groups (217) but these vaccines are not available in South Africa. In the elderly, influenza vaccination has been shown to reduce the incidence of severe disease including bronchopneumonia, hospital admissions and mortality. Trivalent IIV has been shown to provide protection in HIV-infected adults without severe immunosuppression (218). Vaccination of healthcare workers may decrease the risk of spreading influenza to their patients. 
Vaccinating individuals at risk of severe influenza may provide direct protection for these individuals. In addition, vaccinating individuals in close contact with people at risk for severe influenza may provide indirect protection through preventing transmission to high-risk individuals. Groups who should receive influenza vaccine are shown in Box 4.

Box 4: Persons who should receive annual influenza vaccine Pregnant women irrespective of stage of pregnancy, or postpartum HIV-infected adults

Healthcare workers

Persons (adults or children) who are at high risk for influenza and its complications because of underlying medical conditions and who are receiving regular medical care for conditions such as chronic pulmonary (including tuberculosis) and cardiac diseases, chronic renal diseases, diabetes mellitus and similar metabolic disorders, individuals who are immunosuppressed, and individuals who are morbidly obese (body mass index $\geq 40 \mathrm{~kg} / \mathrm{m}^{2}$ )

Residents of old-age homes and chronic care and rehabilitation institutions

Persons aged $\geq 65$ years

Children aged 6-59 months (efficacy of trivalent IIV is low in this group)

Persons aged 6 months to $\leq 18$ years on long-term aspirin therapy

Adults and children who are family contacts of individuals at high risk of severe influenza

Any persons wishing to minimise the risk of influenza acquisition, especially in industrial settings, where large-scale absenteeism could cause significant economic losses

\section{Recommendations}

- All adults $\geq 50$ years who are vaccine naïve should receive a single dose of PCV13 (A II).

- All adults $\geq 50$ who have received PPV23 should receive a single dose of PCV13 one year later (A II).

- All adults $\geq 65$ years who are vaccine naïve should receive a single dose of PCV13 followed a year later by PPV23 (A II).

- All adults $\geq 65$ years who have received PPV23 should receive a single dose of PCV13 at least one year later (A II).

- Younger adults ( $\geq 18$ years) who are vaccine naïve with severe underlying comorbid or immunocompromising conditions including HIV infection should receive a single dose of PCV13 followed at least 2 months later by PPV 23 (A II).

- Younger adults ( $\geq 18$ years) who have previously received PPV23 and have severe underlying comorbid or immunocompromising conditions including HIV infection should receive a single dose of PCV13 one year later (A II).

- All women who are pregnant in the period of influenza vaccine availability (approximately March to June) should be offered influenza vaccination with IIV3 (A II). Adults aged $\geq 65$ years should be offered annual influenza vaccination with IIV3 (A I).

- All adults with specific chronic diseases: chronic pulmonary [including tuberculosis] and cardiac diseases, chronic renal diseases, diabetes mellitus and similar metabolic disorders, individuals who are immunosuppressed including HIVinfected individuals, and individuals who are morbidly obese (body mass index $\geq 40 \mathrm{~kg} / \mathrm{m}^{2}$ ) should be offered annual influenza vaccination with IIV3 (A II).

- All healthcare workers should be offered annual influenza vaccination with IIV3 (A II).

\section{Acknowledgements}

This guideline was developed under the auspices of the South African Thoracic Society and the Federation of Infectious Diseases Societies of Southern Africa. This guideline has been reviewed and endorsed by the Faculty of Consulting Physicians of South Africa, and by Academic Departments of Family Medicine in South Africa. The authors would like to thank Mrs. Rochelle Keene for proof-reading the manuscript. Charles Feldman is supported by the National Research Foundation of South Africa. Keertan Dheda is supported by the South African MRC, EDCTP and South African NRF.

\section{Footnotes}

Conflicts of Interest: AC Whitelaw has received honoraria to the Institute from MSD, Novartis, AstraZeneca, and Takeda. A Brink has received honoraria from MSD, Cipla and Pfizer. C Feldman has acted on the advisory board and/or speakers bureau of companies manufacturing and/or marketing macrolide antibiotics (Abbott, Aspen GSK, Pfizer, Sandoz) and ceftaroline (AstraZeneca/Pfizer) and companies manufacturing and/ or marketing the pneumococcal conjugate vaccine (Pfizer). $C$ Cohen has received grant funds to the Institute from Sanofi and travel paid for by Sanofi and Parexel. GA Richards has received honoraria from Aspen, Cipla, MSD, Pfizer, AstraZeneca, Sanofi, BMS and Fresenius Kabi. K Dheda has received grants, free products/kits from Hain Life Sciences, Alere Diagnostics, FIND Diagnostics, Pfizer, Novartis, Cipla, GSK, AstraZeneca and Sanofi-Aventis. The other authors have no conflicts of interest to declare.

\section{References}

1. Foy HM, Cooney MK, Allan I, et al. Rates of pneumonia during influenza epidemics in Seattle, 1964 to 1975. JAMA 1979;241:253-8. 10.1001/ jama.1979.03290290021018 [PubMed] [Cross Ref]

2. Jokinen $C$, Heiskanen $L$, Juvonen $H$, et al. Incidence of community-acquired pneumonia in the population of four municipalities in eastern Finland. Am J Epidemiol 1993;137:977-88. 10.1093/oxfordjournals.aje.a116770 [PubMed] [Cross Ref]

3. Woodhead MA, Macfarlane JT, McCracken JS, et al. Prospective study of the aetiology and outcome of pneumonia in the community. Lancet 1987;1:671-4. 10.1016/S0140-6736(87)90430-2 [PubMed][Cross Ref]

4. Brink A, Coetzee J, Clay C, et al. The spread of carbapenem-resistant Enterobacteriaceae in South Africa: risk factors for acquisition and prevention. $S$ Afr Med J 2012;102:599-601. 10.7196/SAMJ.5789[PubMed] [Cross Ref]

5. Mandell LA, Wunderink RG, Anzueto A, et al. Infectious Diseases Society of America/American Thoracic Society consensus guidelines on the management of community-acquired pneumonia in adults.Clin Infect Dis 2007;44 Suppl 2:S27-72. 10.1086/511159 [PubMed] [Cross Ref]

6. Lim WS, Baudouin SV, George RC, et al. BTS guidelines for the management of community acquired pneumonia in adults: update 2009. Thorax 2009;64 Suppl 3:iii1-55. 10.1136/thx.2009.121434 [PubMed][Cross Ref]

7. Working Group of the South African Thoracic Society Management of communityacquired pneumonia in adults. S Afr Med J 2007;97:1296-306. [PubMed] 
8. Africa SS. Mortality and causes of death in South Africa, 2013: Findings from death notification P0309.3. Pretoria: Statistics South Africa, 2014.

9. Cohen C, Walaza S, Moyes J, et al. Epidemiology of severe acute respiratory illness (SARI) among adults and children aged $>/=5$ years in a high HIV-prevalence setting, 2009-2012. PLoS One2015;10:e0117716. 10.1371/journal. pone.0117716 [PMC free article] [PubMed] [Cross Ref]

10. Said MA, Johnson HL, Nonyane BA, et al. Estimating the burden of pneumococcal pneumonia among adults: a systematic review and meta-analysis of diagnostic techniques. PLoS One 2013;8:e60273. 10.1371/journal.pone.0060273 [PMC free article] [PubMed] [Cross Ref]

11. von Gottberg A, de Gouveia $L$, Tempia S, et al. Effects of vaccination on invasive pneumococcal disease in South Africa. N Engl J Med 2014;371:1889-99. 10.1056/ NEJMoa1401914 [PubMed][Cross Ref]

12. Cohen C, Dreyer A, Frean J, Treurnich F, lyaloo S, Ismail N, et al. Respiratory pathogens from influenza-like illness and pneumonia surveillance programmes, South Africa 2014. Communicable Diseases Surveillance Bulletin 2015;13:10-30.

13. Kang $\mathrm{Cl}$, Song JH, Oh WS, et al. Clinical outcomes and risk factors of communityacquired pneumonia caused by gram-negative bacilli. Eur J Clin Microbiol Infect Dis 2008;27:657-61. 10.1007/s10096-008-0485-7 [PubMed] [Cross Ref]

14. Pretorius MA, Tempia S, Walaza $S$, et al. The role of influenza, RSV and other common respiratory viruses in severe acute respiratory infections and influenza-like illness in a population with a high HIV sero-prevalence, South Africa 2012-2015. J Clin Virol 2016;75:21-6. 10.1016/j.jcv.2015.12.004 [PMC free article] [PubMed] [Cross Ref]

15. Nyamande K, Lalloo UG, John M. TB presenting as community-acquired pneumonia in a setting of high TB incidence and high HIV prevalence. Int J Tuberc Lung Dis 2007;11:1308-13. [PubMed]

16. Walaza S, Tempia S, Dawood $H$, et al. Influenza virus infection is associated with increased risk of death amongst patients hospitalized with confirmed pulmonary tuberculosis in South Africa, 2010-2011. BMC Infect Dis 2015;15:26015-0746-x. [PMC free article] [PubMed]

17. van Oosterhout JJ, Laufer MK, Graham SM, et al. A community-based study of the incidence of trimethoprim-sulfamethoxazole-preventable infections in Malawian adults living with HIV. J Acquir Immune Defic Syndr 2005;39:626-31. [PubMed]

18. Wasserman S, Engel ME, Griesel R, et al. Burden of pneumocystis pneumonia in HIV-infected adults in sub-Saharan Africa: a systematic review and meta-analysis. BMC Infect Dis 2016;16:482-016-1809-3. [PMC free article] [PubMed]

19. Feldman C, Anderson R. HIV-associated bacterial pneumonia. Clin Chest Med 2013;34:205-16. 10.1016/j.ccm.2013.01.006 [PubMed] [Cross Ref]

20. Al-Tawfiq JA, Zumla $A$, Gautret $P$, et al. Surveillance for emerging respiratory viruses. Lancet Infect Dis 2014;14:992-1000. 10.1016/ S1473-3099(14)70840-0 [PubMed] [Cross Ref]

21. Mandell, Douglas, and Bennett Principles and practice of infectious diseases. Philadelphia: Elsevier, 2010.

22. Huchon GJ, Gialdroni-Grassi G, Leophonte P, et al. Initial antibiotic therapy for lower respiratory tract infection in the community: a European survey. Eur Respir J 1996;9:1590-5. 10.1183/09031936.96.09081590 [PubMed] [Cross Ref]

23. Metlay JP, Stafford RS, Singer DE. National trends in the use of antibiotics by primary care physicians for adult patients with cough. Arch Intern Med 1998;158:1813-8. 10.1001/archinte.158.16.1813 [PubMed][Cross Ref]

24. Diehr P, Wood RW, Bushyhead J, et al. Prediction of pneumonia in outpatients with acute cough--a statistical approach. J Chronic Dis 1984;37:215-25. 10.1016/0021-9681(84)90149-8 [PubMed] [Cross Ref]

25. Snow V, Mottur-Pilson C, Gonzales R. Principles of appropriate antibiotic use for treatment of acute bronchitis in adults. Ann Intern Med 2001;134:518-20. 10.7326/0003-4819-134-6-200103200-00020[PubMed] [Cross Ref]

26. Creer DD, Dilworth JP, Gillespie SH, et al. Aetiological role of viral and bacterial infections in acute adult lower respiratory tract infection (LRTI) in primary care. Thorax 2006;61:75-9. 10.1136/thx.2004.027441 [PMC free article] [PubMed] [Cross Ref]

27. Little $P$, Stuart $B$, Moore $M$, et al. Amoxicillin for acute lower-respiratory-tract infection in primary care when pneumonia is not suspected: a 12-country, randomised, placebo-controlled trial. Lancet Infect Dis 2013;13:123-9. 10.1016/ S1473-3099(12)70300-6 [PubMed] [Cross Ref]

28. Smith SM, Fahey T, Smucny J, et al. Antibiotics for acute bronchitis. Cochrane Database Syst Rev2014;(3):CD000245. [PubMed]
29. Macfarlane J. Lower respiratory tract infection and pneumonia in the community. Semin Respir Infect1999;14:151-62. [PubMed]

30. Butler CC, Hood K, Verheij T, et al. Variation in antibiotic prescribing and its impact on recovery in patients with acute cough in primary care: prospective study in 13 countries. BMJ 2009;338:b2242. 10.1136/bmj.b2242 [PMC free article] [PubMed] [Cross Ref]

31. Little P, Rumsby K, Kelly J, et al. Information leaflet and antibiotic prescribing strategies for acute lower respiratory tract infection: a randomized controlled trial. JAMA 2005;293:3029-35. 10.1001/jama.293.24.3029 [PubMed] [Cross Ref]

32. Aabenhus R, Jensen JU, Jorgensen KJ, et al. Biomarkers as point-of-care tests to guide prescription of antibiotics in patients with acute respiratory infections in primary care. Cochrane Database Syst Rev 2014;(11):CD010130. [PubMed]

33. Brink AJ, Van Wyk J, Moodley VM, et al. The role of appropriate diagnostic testing in acute respiratory tract infections: An antibiotic stewardship strategy to minimise diagnostic uncertainty in primary care. S Afr Med J 2016;106:30-7. 10.7196/SAMJ.2016.v106i6.10857 [PubMed] [Cross Ref]

34. Metlay JP, Fine MJ. Testing strategies in the initial management of patients with community-acquired pneumonia. Ann Intern Med 2003;138:109-18. 10.7326/0003-4819-138-2-200301210-00012 [PubMed][Cross Ref]

35. Metlay JP, Kapoor WN, Fine MJ. Does this patient have communityacquired pneumonia? Diagnosing pneumonia by history and physical examination. JAMA 1997;278:1440-5. 10.1001/ jama.1997.03550170070035 [PubMed] [Cross Ref]

36. O'Brien WT, Sr, Rohweder DA, Lattin GE, Jr, et al. Clinical indicators of radiographic findings in patients with suspected community-acquired pneumonia: who needs a chest x-ray? J Am Coll Radiol2006;3:703-6. 10.1016/j. jacr.2006.02.007 [PubMed] [Cross Ref]

37. Müller B, Harbarth S, Stolz D, et al. Diagnostic and prognostic accuracy of clinical and laboratory parameters in community-acquired pneumonia. BMC Infect Dis 2007;7:10. 10.1186/1471-2334-7-10 [PMC free article] [PubMed] [Cross Ref]

38. Hoare Z, Lim WS. Pneumonia: update on diagnosis and management. BMJ 2006;332:1077-9. 10.1136/bmj.332.7549.1077 [PMC free article] [PubMed] [Cross Ref]

39. Kaplan V, Angus DC, Griffin MF, et al. Hospitalized community-acquired pneumonia in the elderly: age- and sex-related patterns of care and outcome in the United States. Am J Respir Crit Care Med2002;165:766-72. 10.1164/ ajrccm.165.6.2103038 [PubMed] [Cross Ref]

40. Jackson ML, Neuzil KM, Thompson WW, et al. The burden of communityacquired pneumonia in seniors: results of a population-based study. Clin Infect Dis 2004;39:1642-50. 10.1086/425615 [PubMed][Cross Ref]

41. Meehan TP, Fine MJ, Krumholz HM, et al. Quality of care, process, and outcomes in elderly patients with pneumonia. JAMA 1997;278:2080-4. 10.1001/ jama.1997.03550230056037 [PubMed] [Cross Ref]

42. Marrie TJ. Community-acquired pneumonia in the elderly. Clin Infect Dis 2000;31:1066-78. 10.1086/318124 [PubMed] [Cross Ref]

43. Metlay JP, Schulz R, Li YH, et al. Influence of age on symptoms at presentation in patients with community-acquired pneumonia. Arch Intern Med 1997;157:1453 9. 10.1001/archinte.1997.00440340089009 [PubMed] [Cross Ref]

44. Musgrave T, Verghese A. Clinical features of pneumonia in the elderly. Semin Respir Infect1990;5:269-75. [PubMed]

45. Torres A, El-Ebiary M, Riquelme $R$, et al. Community-acquired pneumonia in the elderly. Semin Respir Infect 1999;14:173-83. [PubMed]

46. Riquelme $R$, Torres $A$, el-Ebiary $M$, et al. Community-acquired pneumonia in the elderly. Clinical and nutritional aspects. Am J Respir Crit Care Med 1997;156:1908-14. 10.1164/ajrccm.156.6.9702005[PubMed] [Cross Ref]

47. Fernández-Sabé N, Carratala J, Roson B, et al. Community-acquired pneumonia in very elderly patients: causative organisms, clinical characteristics, and outcomes. Medicine (Baltimore) 2003;82:159-69. 10.1097/01. md.0000076005.64510.87 [PubMed] [Cross Ref]

48. Basi SK, Marrie TJ, Huang JQ, et al. Patients admitted to hospital with suspected pneumonia and normal chest radiographs: epidemiology, microbiology, and outcomes. Am J Med 2004;117:305-11. 10.1016/j. amjmed.2004.03.029 [PubMed] [Cross Ref]

49. Welker JA, Huston M, McCue JD. Antibiotic timing and errors in diagnosing pneumonia. Arch Intern Med 2008;168:351-6. 10.1001/ archinternmed.2007.84 [PubMed] [Cross Ref]

50. Kanwar M, Brar N, Khatib R, et al. Misdiagnosis of community-acquired pneumonia and inappropriate utilization of antibiotics: side effects of the 
4-h antibiotic administration rule. Chest 2007;131:1865-9. 10.1378/chest.070164 [PubMed] [Cross Ref]

51. Pereira JM, Paiva JA, Rello J. Assessing severity of patients with communityacquired pneumonia.Semin Respir Crit Care Med 2012;33:272-83. 10.1055/s0032-1315639 [PubMed] [Cross Ref]

52. Lim WS, van der Eerden MM, Laing R, et al. Defining community acquired pneumonia severity on presentation to hospital: an international derivation and validation study. Thorax 2003;58:377-82. 10.1136/thorax.58.5.377 [PMC free article] [PubMed] [Cross Ref]

53. Kabundji DM, Musekiwa A, Mukansi $M$, et al. Determining need for hospitalisation: Evaluation of the utility of the CRB-65 score in patients with community-acquired pneumonia presenting to an emergency department. $S$ Afr Med J 2014;104:769-72. 10.7196/SAMJ.8150 [Cross Ref]

54. Carratala J, Fernandez-Sabe N, Ortega L, et al. Outpatient care compared with hospitalization for community-acquired pneumonia: a randomized trial in low-risk patients. Ann Intern Med 2005;142:165-72. 10.7326/0003-4819-142-3200502010-00006 [PubMed] [Cross Ref]

55. Guest JF, Morris A. Community-acquired pneumonia: the annual cost to the National Health Service in the UK. Eur Respir J 1997;10:1530-4. 10.1183/09031936.97.10071530 [PubMed] [Cross Ref]

56. Niederman MS, McCombs JS, Unger AN, et al. The cost of treating community-acquired pneumonia.Clin Ther 1998;20:820-37. 10.1016/ S0149-2918(98)80144-6 [PubMed] [Cross Ref]

57. Minnaard MC, de Groot JA, Hopstaken RM, et al. The added value of C-reactive protein measurement in diagnosing pneumonia in primary care: a meta-analysis of individual patient data. CMAJ 2016. [Epub ahead of print]. [PMC free article] [PubMed]

58. Smith RP, Lipworth BJ, Cree IA, et al. C-reactive protein. A clinical marker in community-acquired pneumonia. Chest 1995;108:1288-91. 10.1378/ chest.108.5.1288 [PubMed] [Cross Ref]

59. Chalmers JD, Singanayagam A, Hill AT. C-reactive protein is an independent predictor of severity in community-acquired pneumonia. Am J Med 2008;121:219-25. 10.1016/j.amjmed.2007.10.033 [PubMed][Cross Ref]

60. Schuetz P, Muller B, Christ-Crain M, et al. Procalcitonin to initiate or discontinue antibiotics in acute respiratory tract infections. Cochrane Database Syst Rev 2012;(9):CD007498. [PubMed]

61. Ntusi N, Aubin L, Oliver S, et al. Guideline for the optimal use of blood cultures. S Afr Med J2010;100:839-43. 10.7196/SAMJ.4217 [PubMed] [Cross Ref]

62. Chaisuksant S, Koonsuwan A, Sawanyawisuth K. Appropriateness of obtaining blood cultures in patients with community acquired pneumonia. Southeast Asian J Trop Med Public Health 2013;44:289-94.[PubMed]

63. Campbell SG, Mclvor RA, Joanis V, et al. Can we predict which patients with community-acquired pneumonia are likely to have positive blood cultures? World J Emerg Med 2011;2:272-8. 10.5847/wjem.j.19208642.2011.04.005 [PMC free article] [PubMed] [Cross Ref]

64. Cham G, Yan S, Heng BH, et al. Predicting positive blood cultures in patients presenting with pneumonia at an Emergency Department in Singapore. Ann Acad Med Singap 2009;38:508-7. [PubMed]

65. Falguera M, Trujillano J, Mene R, et al. A Prediction Rule for Estimating the Risk of Bacteremia in Patients with Community-Acquired Pneumonia. Clin Infect Dis 2009;49:409-16. 10.1086/600291[PubMed] [Cross Ref]

66. Benenson RS, Kepner AM, Pyle D, et al. Selective use of blood cultures in emergency department pneumonia patients. J Emerg Med 2007;33:1-8. 10.1016/j.jemermed.2006.12.034 [PubMed] [Cross Ref]

67. Anevlavis S, Petroglou N, Tzavaras A, et al. A prospective study of the diagnostic utility of sputum Gram stain in pneumonia. J Infect 2009;59:83-9. 10.1016/j. jinf.2009.05.011 [PubMed] [Cross Ref]

68. García-Vázquez E, Marcos MA, Mensa J, et al. Assessment of the usefulness of sputum culture for diagnosis of community-acquired pneumonia using the PORT predictive scoring system. Arch Intern Med2004;164:1807-11. 10.1001/ archinte.164.16.1807 [PubMed] [Cross Ref]

69. van $d E$, Vlaspolder $F$, de Graaff $C$, et al. Value of intensive diagnostic microbiological investigation in low- and high-risk patients with communityacquired pneumonia. Eur J Clin Microbiol Infect Dis 2005;24:241-9. [PubMed]

70. Gutiérrez F, Masia $M$, Rodriguez $J C$, et al. Evaluation of the immunochromatographic Binax NOW assay for detection of Streptococcus pneumoniae urinary antigen in a prospective study of community-acquired pneumonia in Spain. Clin Infect Dis 2003;36:286-92. 10.1086/345852 [PubMed] [Cross Ref]

71. Harris AM, Beekmann SE, Polgreen PM, et al. Rapid urine antigen testing for Streptococcus pneumoniae in adults with community-acquired pneumonia: clinical use and barriers. Diagn Microbiol Infect Dis 2014;79:454-7. 10.1016/j. diagmicrobio.2014.05.008 [PubMed] [Cross Ref]

72. Piso RJ, Iven-Koller D, Koller MT, et al. The routine use of urinary pneumococcal antigen test in hospitalised patients with community acquired pneumonia has limited impact for adjustment of antibiotic treatment. Swiss Med Wkly 2012;142:w13679-9. [PubMed]

73. Dunn JJ, Ginocchio CC. Can newly developed, rapid immunochromatographic antigen detection tests be reliably used for the laboratory diagnosis of influenza virus infections? J Clin Microbiol 2015;53:1790-6. 10.1128/JCM.02739-14 [PMC free article] [PubMed] [Cross Ref]

74. Liu YF, Gao Y, Chen M, et al. Etiological analysis and predictive diagnostic model building of community-acquired pneumonia in adult outpatients in Beijing, China. BMC Infect Dis 2013;13:309-9. 10.1186/1471-2334-13-309 [PMC free article] [PubMed] [Cross Ref]

75. Huijskens EG, Rossen JW, Kluytmans JA, et al. Evaluation of yield of currently available diagnostics by sample type to optimize detection of respiratory pathogens in patients with a community-acquired pneumonia. Influenza Other Respir Viruses 2014;8:243-9. 10.1111/irv.12153 [PMC free article] [PubMed][Cross Ref]

76. Corbett EL, Watt CJ, Walker N, et al. The growing burden of tuberculosis: global trends and interactions with the HIV epidemic. Arch Intern Med 2003;163:100921. 10.1001/archinte.163.9.1009[PubMed] [Cross Ref]

77. Organization WH. Global tuberculosis report. 2014.

78. Dooley KE, Chaisson RE. Tuberculosis and diabetes mellitus: convergence of two epidemics. Lancet Infect Dis 2009;9:737-46. 10.1016/ S1473-3099(09)70282-8 [PMC free article] [PubMed] [Cross Ref]

79. Pizzol D, Di Gennaro F, Chhaganlal KD, et al. Tuberculosis and diabetes: current state and future perspectives. Trop Med Int Health 2016;21:694-702. 10.1111/ tmi.12704 [PubMed] [Cross Ref]

80. Calligaro GL, Theron $\mathrm{G}$, Khalfey $\mathrm{H}$, et al. Burden of tuberculosis in intensive care units in Cape Town, South Africa, and assessment of the accuracy and effect on patient outcomes of the Xpert MTB/RIF test on tracheal aspirate samples for diagnosis of pulmonary tuberculosis: a prospective burden of disease study with a nested randomised controlled trial. Lancet Respir Med 2015;3:621-30. 10.1016/ S2213-2600(15)00198-8 [PubMed] [Cross Ref]

81. Steingart KR, Henry M, Ng V, et al. Fluorescence versus conventional sputum smear microscopy for tuberculosis: a systematic review. Lancet Infect Dis 2006;6:570-81. 10.1016/S1473-3099(06)70578-3[PubMed] [Cross Ref]

82. Steingart KR, Schiller I, Horne DJ, et al. Xpert $\hat{A}^{\oplus}$ MTB/RIF assay for pulmonary tuberculosis and rifampicin resistance in adults. Cochrane Database Syst Rev 2014;(1):CD009593. [PMC free article][PubMed]

83. Peter JG, Theron G, Pooran A, et al. Comparison of two methods for acquisition of sputum samples for diagnosis of suspected tuberculosis in smear-negative or sputum-scarce people: a randomised controlled trial. Lancet Respir Med 2013;1:471-8. 10.1016/S2213-2600(13)70120-6 [PMC free article] [PubMed] [Cross Ref]

84. Wang SF, Ou XC, Li Q, et al. The Abbott RealTime MTB assay and the Cepheid GeneXpert assay show comparable performance for the detection of Mycobacterium tuberculosis in sputum specimens. Int J Infect Dis 2016;45:7880. 10.1016/j.jijid.2016.02.024 [PubMed] [Cross Ref]

85. Seifert M, Ajbani K, Georghiou SB, et al. A performance evaluation of MTBDRplus version 2 for the diagnosis of multidrug-resistant tuberculosis. Int J Tuberc Lung Dis 2016;20:631-7. 10.5588/ijtld.15.0788 [PMC free article] [PubMed] [Cross Ref]

86. World Health Organization. The use of molecular line probe assays for the detection of mutations associated with resistance to fluoroquinolones (FQs) and second-line injectable drugs (SLIDs). Policy Guidance 2016.

87. Shah $M$, Variava $E$, Holmes $C B$, et al. Diagnostic accuracy of a urine lipoarabinomannan test for tuberculosis in hospitalized patients in a High HIV prevalence setting. J Acquir Immune Defic Syndr2009;52:145-51. 10.1097/ QAl.0b013e3181b98430 [PMC free article] [PubMed] [Cross Ref]

88. Peter JG, Theron G, van Zyl-Smit R, et al. Diagnostic accuracy of a urine lipoarabinomannan strip-test for TB detection in HIV-infected hospitalised patients. Eur Respir J 2012;40:1211-20. 10.1183/09031936.00201711 [PMC free article] [PubMed] [Cross Ref] 
89. Peter JG, Theron G, Dheda K. Can point-of-care urine LAM strip testing for tuberculosis add value to clinical decision making in hospitalised HIV-infected persons? PLoS One 2013;8:e54875. 10.1371/journal.pone.0054875 [PMC free article] [PubMed] [Cross Ref]

90. Peter JG, Zijenah LS, Chanda D, et al. Effect on mortality of point-of-care, urine-based lipoarabinomannan testing to guide tuberculosis treatment initiation in HIV-positive hospital inpatients: a pragmatic, parallel-group, multicountry, open-label, randomised controlled trial. Lancet 2016;387:1187-97. 10.1016/S0140-6736(15)01092-2 [PubMed] [Cross Ref]

91. Thomas CF, Jr, Limper AH. Pneumocystis pneumonia. N Engl J Med 2004;350:2487-98. 10.1056/NEJMra032588 [PubMed] [Cross Ref]

92. Cruciani M, Marcati $P$, Malena $M$, et al. Meta-analysis of diagnostic procedures for Pneumocystis carinii pneumonia in HIV-1-infected patients. Eur Respir J 2002;20:982-9. 10.1183/09031936.02.01372002[PubMed] [Cross Ref]

93. Wakefield $A E$, Guiver $L$, Miller RF, et al. DNA amplification on induced sputum samples for diagnosis of Pneumocystis carinii pneumonia. Lancet 1991;337:1378-9. 10.1016/0140-6736(91)93062-E [PubMed] [Cross Ref]

94. Lipschik GY, Gill VJ, Lundgren JD, et al. Improved diagnosis of Pneumocystis carinii infection by polymerase chain reaction on induced sputum and blood. Lancet 1992;340:203-6. 10.1016/0140-6736(92)90469-J [PubMed] [Cross Ref]

95. Roux P, Lavrard I, Poirot JL, et al. Usefulness of PCR for detection of Pneumocystis carinii DNA. J Clin Microbiol 1994;32:2324-6. [PMC free article] [PubMed]

96. Flori $P$, Bellete $B$, Durand $F$, et al. Comparison between real-time PCR, conventional $P C R$ and different staining techniques for diagnosing Pneumocystis jiroveci pneumonia from bronchoalveolar lavage specimens. J Med Microbiol 2004;53:603-7. 10.1099/jmm.0.45528-0 [PubMed] [Cross Ref]

97. Harris JR, Marston BJ, Sangrujee N, et al. Cost-effectiveness analysis of diagnostic options for pneumocystis pneumonia (PCP). PLoS One 2011;6:e23158. 10.1371/ journal.pone.0023158 [PMC free article] [PubMed] [Cross Ref]

98. McTaggart LR, Wengenack NL, Richardson SE. Validation of the MycAssay Pneumocystis kit for detection of Pneumocystis jirovecii in bronchoalveolar lavage specimens by comparison to a laboratory standard of direct immunofluorescence microscopy, real-time $P C R$, or conventional PCR. J Clin Microbiol2012;50:1856-9. 10.1128/JCM.05880-11 [PMC free article] [PubMed] [Cross Ref]

99. Hauser PM, Bille J, Lass-Florl C, et al. Multicenter, prospective clinical evaluation of respiratory samples from subjects at risk for Pneumocystis jirovecii infection by use of a commercial real-time PCR assay. J Clin Microbiol 2011;49:1872-8. 10.1128/JCM.02390-10 [PMC free article] [PubMed] [Cross Ref]

100. Wood BR, Komarow L, Zolopa AR, et al. Test performance of blood beta-glucan for Pneumocystis jirovecii pneumonia in patients with AIDS and respiratory symptoms. Aids 2013;27:967-72. 10.1097/QAD.0b013e32835cb646 [PMC free article] [PubMed] [Cross Ref]

101. Zaman MK, White DA. Serum lactate dehydrogenase levels and Pneumocystis carinii pneumonia. Diagnostic and prognostic significance. Am Rev Respir Dis 1988;137:796-800. 10.1164/ajrccm/137.4.796[PubMed] [Cross Ref]

102. Quist J, Hill AR. Serum lactate dehydrogenase (LDH) in Pneumocystis carinii pneumonia, tuberculosis, and bacterial pneumonia. Chest 1995;108:415-8. 10.1378/chest.108.2.415 [PubMed][Cross Ref]

103. WHO. WHO case definitions of HIV for surveillance and revised clinical staging and immunological classification of HIV-related disease in adults and children. Geneva, Switzerland, 2007.

104. Pakhale S, Mulpuru S, Verheij TJ, et al. Antibiotics for communityacquired pneumonia in adult outpatients. Cochrane Database Syst Rev 2014;(10):CD002109. [PubMed]

105. Ruiz M, Ewig S, Marcos MA, et al. Etiology of community-acquired pneumonia: impact of age, comorbidity, and severity. Am J Respir Crit Care Med 1999;160:397-405. 10.1164/ajrccm.160.2.9808045[PubMed] [Cross Ref]

106. El-Solh AA, Sikka P, Ramadan F, et al. Etiology of severe pneumonia in the very elderly. Am J Respir Crit Care Med 2001;163:645-51. 10.1164/ ajrccm.163.3.2005075 [PubMed] [Cross Ref]

107. Cillóniz C, Polverino E, Ewig S, et al. Impact of age and comorbidity on cause and outcome in community-acquired pneumonia. Chest 2013;144:999-1007. 10.1378/chest.13-0062 [PubMed] [Cross Ref]

108. Niederman MS. In the Clinic: Community-Acquired Pneumonia. Ann Intern Med 2015;163:ITC1-17. 10.7326/AITC201510060 [PubMed] [Cross Ref]
109. Belforti RK, Lagu T, Haessler S, et al. Association Between Initial Route of Fluoroquinolone Administration and Outcomes in Patients Hospitalized for Community-acquired Pneumonia. Clin Infect Dis 2016;63:1-9. 10.1093/cid/ ciw209 [PMC free article] [PubMed] [Cross Ref]

110. Gattarello S, Borgatta B, Sole-Violan J, et al. Decrease in mortality in severe community-acquired pneumococcal pneumonia: impact of improving antibiotic strategies (2000-2013). Chest 2014;146:22-31. 10.1378/chest.131531 [PubMed] [Cross Ref]

111. Gattarello S, Lagunes L, Vidaur L, et al. Improvement of antibiotic therapy and ICU survival in severe non-pneumococcal community-acquired pneumonia: a matched case-control study. Crit Care 2015;19:335-015-1051-1. [PMC free article] [PubMed]

112. Sligl WI, Asadi L, Eurich DT, et al. Macrolides and mortality in critically ill patients with community-acquired pneumonia: a systematic review and meta-analysis. Crit Care Med 2014;42:420-32. 10.1097/ CCM.0b013e3182a66b9b [PubMed] [Cross Ref]

113. Lee JS, Giesler DL, Gellad WF, et al. Antibiotic Therapy for Adults Hospitalized With Community-Acquired Pneumonia: A Systematic Review. JAMA 2016;315:593-602. 10.1001/jama.2016.0115[PubMed] [Cross Ref]

114. Martin-Loeches I, Lisboa T, Rodriguez A, et al. Combination antibiotic therapy with macrolides improves survival in intubated patients with communityacquired pneumonia. Intensive Care Med2010;36:612-20. 10.1007/s00134009-1730-y [PubMed] [Cross Ref]

115. Restrepo MI, Mortensen EM, Waterer GW, et al. Impact of macrolide therapy on mortality for patients with severe sepsis due to pneumonia. Eur Respir J 2009;33:153-9. 10.1183/09031936.00054108 [PubMed][Cross Ref]

116. File TM, Garau J, Jacobs MR, et al. Efficacy of a new pharmacokinetically enhanced formulation of amoxicillin/clavulanate $(2000 / 125 \mathrm{mg})$ in adults with community-acquired pneumonia caused by Streptococcus pneumoniae, including penicillin-resistant strains. Int J Antimicrob Agents 2005;25:110-9. 10.1016/j.ijantimicag.2004.10.007 [PubMed] [Cross Ref]

117. File TM, Jr, Low DE, Eckburg PB, et al. Integrated analysis of FOCUS 1 and FOCUS 2: randomized, doubled-blinded, multicenter phase 3 trials of the efficacy and safety of ceftaroline fosamil versus ceftriaxone in patients with community-acquired pneumonia. Clin Infect Dis 2010;51:1395-405 10.1086/657313 [PubMed] [Cross Ref]

118. Ortiz-Ruiz G, Vetter $N$, Isaacs $R$, et al. Ertapenem versus ceftriaxone for the treatment of community-acquired pneumonia in adults: combined analysis of two multicentre randomized, double-blind studies. J Antimicrob Chemother 2004;53 Suppl 2:ii59-66. 10.1093/jac/dkh207 [PubMed] [Cross Ref]

119. Muthuri SG, Venkatesan S, Myles PR, et al. Effectiveness of neuraminidase inhibitors in reducing mortality in patients admitted to hospital with influenza A H1N1pdm09 virus infection: a meta-analysis of individual participant data. Lancet Respir Med 2014;2:395-404. 10.1016/S2213-2600(14)700414[PubMed] [Cross Ref]

120. Noel ZR, Bastin ML, Montgomery AA, et al. Comparison of High-Dose Versus Standard Dose Oseltamivir in Critically III Patients With Influenza. J Intensive Care Med 2016. [Epub ahead of print]. 10.1177/0885066616638649 [PubMed] [Cross Ref]

121. NICD \& NHLS. Healthcare Workers Handbook on Influenza 2015.

122. Van Kerkhove MD, Vandemaele KA, Shinde V, et al. Risk factors for severe outcomes following 2009 influenza A (H1N1) infection: a global pooled analysis. PLoS Med 2011;8:e1001053. 10.1371/journal.pmed.1001053 [PMC free article] [PubMed] [Cross Ref]

123. Abadom TR, Smith AD, Tempia S, et al. Risk factors associated with hospitalisation for influenza-associated severe acute respiratory illness in South Africa: A case-population study. Vaccine 2016;34:5649-55. 10.1016/j. vaccine.2016.09.011 [PMC free article] [PubMed] [Cross Ref]

124. Wunderink RG, Mandell L. Adjunctive therapy in community-acquired pneumonia. Semin Respir Crit Care Med 2012;33:311-8. 10.1055/s-00321315643 [PubMed] [Cross Ref]

125. Meijvis SC, van de Garde EM, Rijkers GT, et al. Treatment with anti-inflammatory drugs in community-acquired pneumonia. J Intern Med 2012;272:25-35. 10.1111/j.1365-2796.2012.02554.x[PubMed] [Cross Ref]

126. Morton B, Pennington SH, Gordon SB. Immunomodulatory adjuvant therapy in severe community-acquired pneumonia. Expert Rev Respir Med 2014;8:58796. 10.1586/17476348.2014.927736 [PubMed][Cross Ref] 
127. Chopra V, Rogers MA, Buist $M$, et al. Is statin use associated with reduced mortality after pneumonia? A systematic review and meta-analysis. Am J Med 2012;125:1111-23. 10.1016/j.amjmed.2012.04.011 [PubMed] [Cross Ref]

128. Troeman DP, Postma DF, van Werkhoven $\mathrm{CH}$, et al. The immunomodulatory effects of statins in community-acquired pneumonia: a systematic review. J Infect 2013;67:93-101. 10.1016/j.jinf.2013.04.015[PubMed] [Cross Ref]

129. Khan AR, Riaz M, Bin Abdulhak AA, et al. The role of statins in prevention and treatment of community acquired pneumonia: a systematic review and meta-analysis. PLoS One 2013;8:e52929. 10.1371/journal.pone.0052929 [PMC free article] [PubMed] [Cross Ref]

130. Fitzgerald $E$, Duquette $L$, Williams M. Established statin use reduces mortality from community-acquired pneumonia:a systematic review and meta-analysis. EMJ Respir 2015;3:46-55.

131. Viasus D, Garcia-Vidal C, Simonetti AF, et al. The effect of simvastatin on inflammatory cytokines in community-acquired pneumonia: a randomised, double-blind, placebo-controlled trial. BMJ Open 2015;5:e006251-2014006251. [PMC free article] [PubMed]

132. Horita N, Otsuka T, Haranaga S, et al. Adjunctive Systemic Corticosteroids for Hospitalized Community-Acquired Pneumonia: Systematic Review and Meta-Analysis 2015 Update. Sci Rep2015;5:14061. 10.1038/srep14061 [PMC free article] [PubMed] [Cross Ref]

133. Tagami T, Matsui $H$, Horiguchi $H$, et al. Low-dose corticosteroid use and mortality in severe community-acquired pneumonia patients. Eur Respir J 2015;45:463-72. 10.1183/09031936.00081514[PubMed] [Cross Ref]

134. Blum CA, Nigro N, Briel M, et al. Adjunct prednisone therapy for patients with community-acquired pneumonia: a multicentre, double-blind, randomised, placebo-controlled trial. Lancet 2015;385:1511-8. 10.1016/ S0140-6736(14)62447-8 [PubMed] [Cross Ref]

135. Torres A, Sibila O, Ferrer M, et al. Effect of corticosteroids on treatment failure among hospitalized patients with severe community-acquired pneumonia and high inflammatory response: a randomized clinical trial. JAMA 2015;313:677-86. 10.1001/jama.2015.88 [PubMed] [Cross Ref]

136. Chen LP, Chen JH, Chen Y, et al. Efficacy and safety of glucocorticoids in the treatment of community-acquired pneumonia: A meta-analysis of randomized controlled trials. World J Emerg Med2015;6:172-8. 10.5847/wjem.j.19208642.2015.03.002 [PMC free article] [PubMed] [Cross Ref]

137. Marti C, Grosgurin O, Harbarth S, et al. Adjunctive Corticotherapy for Community Acquired Pneumonia: A Systematic Review and Meta-Analysis. PLoS One 2015;10:e0144032. 10.1371/journal. pone.0144032 [PMC free article] [PubMed] [Cross Ref]

138. Siemieniuk RA, Meade MO, Alonso-Coello P, et al. Corticosteroid Therapy for Patients Hospitalized With Community-Acquired Pneumonia: A Systematic Review and Meta-analysis. Ann Intern Med2015;163:519-28. 10.7326/ M15-0715 [PubMed] [Cross Ref]

139. Wan YD, Sun TW, Liu ZQ, et al. Efficacy and Safety of Corticosteroids for Community-Acquired Pneumonia: A Systematic Review and Meta-Analysis. Chest 2016;149:209-19. 10.1378/chest.15-1733[PubMed] [Cross Ref]

140. Feldman C, Anderson R. Corticosteroids in the adjunctive therapy of community-acquired pneumonia: an appraisal of recent meta-analyses of clinical trials. J Thorac Dis 2016;8:E162-71. 10.21037/jtd.2016.02.43 [PMC free article] [PubMed] [Cross Ref]

141. Popovic M, Blum CA, Nigro $N$, et al. Benefit of adjunct corticosteroids for community-acquired pneumonia in diabetic patients. Diabetologia 2016;59:2552-60. 10.1007/s00125-0164091-4 [PubMed][Cross Ref]

142. Fine MJ, Smith MA, Carson CA, et al. Prognosis and outcomes of patients with community-acquired pneumonia. A meta-analysis. JAMA 1996;275:134-41. 10.1001/jama.1996.03530260048030 [PubMed][Cross Ref]

143. Sligl WI, Eurich DT, Marrie TJ, et al. Age still matters: prognosticating shortand long-term mortality for critically ill patients with pneumonia. Crit Care Med 2010;38:2126-32. 10.1097/CCM.0b013e3181eedaeb [PubMed] [Cross Ref]

144. Dellinger RP, Levy MM, Rhodes A, et al. Surviving Sepsis Campaign: international guidelines for management of severe sepsis and septic shock, 2012. Intensive Care Med 2013;39:165-228. 10.1007/s00134-0122769-8 [PubMed] [Cross Ref]

145. Amato MB, Meade MO, Slutsky AS, et al. Driving pressure and survival in the acute respiratory distress syndrome. N Engl J Med 2015;372:747-55. 10.1056/ NEJMsa1410639 [PubMed] [Cross Ref]
146. Eliakim-Raz N, Robenshtok E, Shefet D, et al. Empiric antibiotic coverage of atypical pathogens for community-acquired pneumonia in hospitalized adults. Cochrane Database Syst Rev 2012;(9):CD004418.[PubMed]

147. Muthuri SG, Venkatesan S, Myles PR, et al. Impact of neuraminidase inhibitors on influenza $A(\mathrm{H} 1 \mathrm{~N} 1) p d m 09-$-related pneumonia: an individual participant data meta-analysis. Influenza Other Respir Viruses 2016;10:192-204. 10.1111/ irv.12363 [PMC free article] [PubMed] [Cross Ref]

148. Bruns $A H$, Oosterheert JJ, Hak $E$, et al. Usefulness of consecutive C-reactive protein measurements in follow-up of severe communityacquired pneumonia. Rev Port Pneumol 2009;15:129-32. 10.1016/ S0873-2159(15)30118-5 [PubMed] [Cross Ref]

149. Nathwani $D$, Lawson $W$, Dryden $M$, et al. Implementing criteria-based early switch/early discharge programmes: a European perspective. Clin Microbiol Infect 2015;21 Suppl 2:S47-55. 10.1016/j.cmi.2015.03.023 [PubMed] [Cross Ref]

150. Oosterheert JJ, Bonten MJ, Schneider MM, et al. Effectiveness of early switch from intravenous to oral antibiotics in severe community acquired pneumonia: multicentre randomised trial. BMJ2006;333:1193. 10.1136/ bmj.38993.560984.BE [PMC free article] [PubMed] [Cross Ref]

151. Engel MF, Bruns $A H, H u l s c h e r ~ M E$, et al. A tailored implementation strategy to reduce the duration of intravenous antibiotic treatment in communityacquired pneumonia: a controlled before-and-after study.Eur J Clin Microbiol Infect Dis 2014;33:1897-908. 10.1007/s10096-014-2158-z [PubMed] [Cross Ref]

152. Light RW, Girard WM, Jenkinson SG, et al. Parapneumonic effusions. Am J Med 1980;69:507-12. 10.1016/0002-9343(80)90460-X [PubMed] [Cross Ref]

153. Light RW, Macgregor Ml, Luchsinger PC, et al. Pleural effusions: the diagnostic separation of transudates and exudates. Ann Intern Med 1972;77:507-13. 10.7326/0003-4819-77-4-507 [PubMed][Cross Ref]

154. Wilcox ME, Chong CA, Stanbrook MB, et al. Does this patient have an exudative pleural effusion? The Rational Clinical Examination systematic review. JAMA 2014;311:2422-31. 10.1001/jama.2014.5552[PubMed] [Cross Ref]

155. Good JT, Jr, Taryle DA, Maulitz RM, et al. The diagnostic value of pleural fluid pH. Chest1980;78:55-9. 10.1378/chest.78.1.55 [PubMed] [Cross Ref]

156. Houston MC. Pleural fluid $\mathrm{pH}$ : diagnostic, therapeutic, and prognostic value. Am J Surg1987;154:333-7. 10.1016/0002-9610(89)90623-5 [PubMed] [Cross Ref]

157. Seedat MA, Feldman C, Skoularigis J, et al. A study of acute communityacquired pneumonia, including details of cardiac changes. Q J Med 1993;86:669-75. 10.1093/qjmed/86.10.669 [PubMed][Cross Ref]

158. Musher DM, Rueda AM, Kaka AS, et al. The association between pneumococcal pneumonia and acute cardiac events. Clin Infect Dis 2007;45:158-65. 10.1086/518849 [PubMed] [Cross Ref]

159. Ramirez J, Aliberti S, Mirsaeidi M, et al. Acute myocardial infarction in hospitalized patients with community-acquired pneumonia. Clin Infect Dis 2008;47:182-7. 10.1086/589246 [PubMed] [Cross Ref]

160. Corrales-Medina VF, Suh KN, Rose G, et al. Cardiac complications in patients with community-acquired pneumonia: a systematic review and meta-analysis of observational studies. PLoS Med2011;8:e1001048. 10.1371/journal. pmed.1001048 [PMC free article] [PubMed] [Cross Ref]

161. Corrales-Medina VF, Musher DM, Wells GA, et al. Cardiac complications in patients with community-acquired pneumonia: incidence, timing, risk factors, and association with short-term mortality.Circulation 2012;125:773-81. 10.1161/CIRCULATIONAHA.111.040766 [PubMed] [Cross Ref]

162. Corrales-Medina VF, Musher DM, Shachkina $S$, et al. Acute pneumonia and the cardiovascular system. Lancet 2013;381:496-505. 10.1016/ S0140-6736(12)61266-5 [PubMed] [Cross Ref]

163. Soto-Gomez N, Anzueto A, Waterer GW, et al. Pneumonia: an arrhythmogenic disease? Am J Med2013;126:43-8. 10.1016/j.amjmed.2012.08.005 [PMC free article] [PubMed] [Cross Ref]

164. Cangemi R, Casciaro M, Rossi $E$, et al. Platelet activation is associated with myocardial infarction in patients with pneumonia. J Am Coll Cardiol 2014;64:1917-25. 10.1016/j.jacc.2014.07.985 [PubMed][Cross Ref]

165. Feldman C, Anderson R. Community-Acquired Pneumonia: Pathogenesis of Acute Cardiac Events and Potential Adjunctive Therapies. Chest 2015;148:523 32. 10.1378/chest.15-0484 [PubMed] [Cross Ref]

166. Falcone $M$, Russo $A$, Cangemi $R$, et al. Lower mortality rate in elderly patients with community-onset pneumonia on treatment with aspirin. J Am Heart Assoc 2015;4:e001595. 10.1161/JAHA.114.001595 [PMC free article] [PubMed] [Cross Ref] 
167. Brown AO, Mann B, Gao G, et al. Streptococcus pneumoniae translocates into the myocardium and forms unique microlesions that disrupt cardiac function. PLoS Pathog 2014;10:e1004383. 10.1371/journal.ppat.1004383 [PMC free article] [PubMed] [Cross Ref]

168. Brown AO, Millett ER, Quint JK, et al. Cardiotoxicity during invasive pneumococcal disease. Am J Respir Crit Care Med 2015;191:739-45. 10.1164/ rccm.201411-1951PP [PMC free article] [PubMed][Cross Ref]

169. Viasus D, Garcia-Vidal C, Manresa F, et al. Risk stratification and prognosis of acute cardiac events in hospitalized adults with community-acquired pneumonia. J Infect 2013;66:27-33. 10.1016/j.jinf.2012.09.003 [PubMed] [Cross Ref]

170. Corrales-Medina VF, Alvarez KN, Weissfeld LA, et al. Association between hospitalization for pneumonia and subsequent risk of cardiovascular disease. JAMA 2015;313:264-74. 10.1001/jama.2014.18229 [PMC free article] [PubMed] [Cross Ref]

171. Cangemi R, Calvieri C, Falcone M, et al. Relation of Cardiac Complications in the Early Phase of Community-Acquired Pneumonia to Long-Term Mortality and Cardiovascular Events. Am J Cardiol2015;116:647-51. 10.1016/j. amjcard.2015.05.028 [PubMed] [Cross Ref]

172. Feldman C, Anderson R. Community-acquired pneumonia: still a major burden of disease. Curr Opin Crit Care 2016;22:477-84. 10.1097/ MCC.0000000000000340 [PubMed] [Cross Ref]

173. Marik PE. Aspiration pneumonitis and aspiration pneumonia. N Engl J Med 2001;344:665-71. 10.1056/NEJM200103013440908 [PubMed] [Cross Ref]

174. Lode H. Microbiological and clinical aspects of aspiration pneumonia. J Antimicrob Chemother 1988;21 Suppl C:83-90. [PubMed]

175. Bartlett JG, Gorbach SL. The triple threat of aspiration pneumonia. Chest 1975;68:560-6. 10.1378/chest.68.4.560 [PubMed] [Cross Ref]

176. Finegold SM. Aspiration pneumonia. Rev Infect Dis 1991;13 Suppl 9:S737-42. 10.1093/clinids/13.Supplement_9.S737 [PubMed] [Cross Ref]

177. Tuomanen El, Austrian R, Masure HR. Pathogenesis of pneumococcal infection. N Engl J Med1995;332:1280-4. 10.1056/ NEJM199505113321907 [PubMed] [Cross Ref]

178. Mullan H, Roubenoff RA, Roubenoff R. Risk of pulmonary aspiration among patients receiving enteral nutrition support. JPEN J Parenter Enteral Nutr 1992;16:160-4. 10.1177/0148607192016002160[PubMed] [Cross Ref]

179. DiBardino DM, Wunderink RG. Aspiration pneumonia: a review of modern trends. J Crit Care2015;30:40-8. 10.1016/j.jcrc.2014.07.011 [PubMed] [Cross Ref]

180. Lipper B, Simon D, Cerrone F. Pulmonary aspiration during emergency endoscopy in patients with upper gastrointestinal hemorrhage. Crit Care Med 1991;19:330-3. 10.1097/00003246-199103000-00008[PubMed] [Cross Ref]

181. Rudolph SJ, Landsverk BK, Freeman ML. Endotracheal intubation for airway protection during endoscopy for severe upper GI hemorrhage. Gastrointest Endosc 2003;57:58-61. 10.1067/mge.2003.46[PubMed] [Cross Ref]

182. Reza Shariatzadeh M, Huang JQ, Marrie TJ. Differences in the features of aspiration pneumonia according to site of acquisition: community or continuing care facility. J Am Geriatr Soc 2006;54:296-302. 10.1111/j.15325415.2005.00608.x [PubMed] [Cross Ref]

183. Marrie TJ, Durant H, Yates L. Community-acquired pneumonia requiring hospitalization: 5-year prospective study. Rev Infect Dis 1989;11:586-99. 10.1093/clinids/11.4.586 [PubMed] [Cross Ref]

184. Torres A, Serra-Batlles J, Ferrer A, et al. Severe community-acquired pneumonia. Epidemiology and prognostic factors. Am Rev Respir Dis 1991;144:312-8. 10.1164/ajrccm/144.2.312 [PubMed] [Cross Ref]

185. Moine $P$, Vercken JB, Chevret $S$, et al. Severe community-acquired pneumonia. Etiology, epidemiology, and prognosis factors. French Study Group for Community-Acquired Pneumonia in the Intensive Care Unit. Chest 1994;105:1487-95. 10.1378/chest.105.5.1487 [PubMed] [Cross Ref]

186. Taylor JK, Fleming GB, Singanayagam A, et al. Risk factors for aspiration in community-acquired pneumonia: analysis of a hospitalized UK cohort. Am J Med 2013;126:995-1001. 10.1016/j.amjmed.2013.07.012 [PubMed] [Cross Ref]

187. Holas MA, DePippo KL, Reding MJ. Aspiration and relative risk of medical complications following stroke. Arch Neurol 1994;51:1051-3. 10.1001/ archneur.1994.00540220099020 [PubMed] [Cross Ref]
188. Westendorp WF, Nederkoorn PJ, Vermeij JD, et al. Post-stroke infection: a systematic review and meta-analysis. BMC Neurol 2011;11:110. 10.1186/14712377-11-110 [PMC free article] [PubMed][Cross Ref]

189. Leroy O, Vandenbussche C, Coffinier C, et al. Community-acquired aspiration pneumonia in intensive care units. Epidemiological and prognosis data. Am J Respir Crit Care Med 1997;156:1922-9. 10.1164/ ajrccm.156.6.9702069 [PubMed] [Cross Ref]

190. Leroy O, Santre C, Beuscart C, et al. A five-year study of severe communityacquired pneumonia with emphasis on prognosis in patients admitted to an intensive care unit. Intensive Care Med 1995;21:24-31. 10.1007/ BF02425150 [PubMed] [Cross Ref]

191. Kozlow JH, Berenholtz SM, Garrett $E$, et al. Epidemiology and impact of aspiration pneumonia in patients undergoing surgery in Maryland, 1999-2000. Crit Care Med 2003;31:1930-7. 10.1097/01. CCM.0000069738.73602.5F [PubMed] [Cross Ref]

192. Adnet F, Baud F. Relation between Glasgow Coma Scale and aspiration pneumonia. Lancet1996;348:123-4. 10.1016/ S0140-6736(05)64630-2 [PubMed] [Cross Ref]

193. Mokhlesi B, Logemann JA, Rademaker AW, et al. Oropharyngeal deglutition in stable COPD. Chest2002;121:361-9. 10.1378/chest.121.2.361 [PubMed] [Cross Ref]

194. Nosè $M$, Recla $E$, Trifirò $G$, et al. Antipsychotic drug exposure and risk of pneumonia: a systematic review and meta-analysis of observational studies. Pharmacoepidemiol Drug Saf 2015;24:812-20. 10.1002/ pds.3804 [PubMed] [Cross Ref]

195. Terpenning MS, Taylor GW, Lopatin DE, et al. Aspiration pneumonia: dental and oral risk factors in an older veteran population. J Am Geriatr Soc 2001;49:55763. 10.1046/j.1532-5415.2001.49113.x[PubMed] [Cross Ref]

196. Scannapieco FA, Papandonatos GD, Dunford RG. Associations between oral conditions and respiratory disease in a national sample survey population. Ann Periodontol 1998;3:251-6. 10.1902/ annals.1998.3.1.251 [PubMed] [Cross Ref]

197. Park RH, Allison MC, Lang J, et al. Randomised comparison of percutaneous endoscopic gastrostomy and nasogastric tube feeding in patients with persisting neurological dysphagia. BMJ 1992;304:1406-9. 10.1136/ bmj.304.6839.1406 [PMC free article] [PubMed] [Cross Ref]

198. Bartlett JG. How important are anaerobic bacteria in aspiration pneumonia: when should they be treated and what is optimal therapy. Infect Dis Clin North Am 2013;27:149-55. 10.1016/j.idc.2012.11.016[PubMed] [Cross Ref]

199. Bartlett JG. Anaerobic bacterial infections of the lung and pleural space. Clin Infect Dis 1993;16Suppl 4:S248-55. 10.1093/clinids/16.Supplement_4. S248 [PubMed] [Cross Ref]

200. Finegold SM, George WL, Mulligan ME. Anaerobic infections. Part I. Dis Mon 1985;31:1-77.[PubMed]

201. Moser KM, Maurer J, Jassy L, et al. Sensitivity, specificity, and risk of diagnostic procedures in a canine model of Streptococcus pneumoniae pneumonia. Am Rev Respir Dis 1982;125:436-42. [PubMed]

202. Mier L, Dreyfuss D, Darchy B, et al. Is penicillin $G$ an adequate initial treatment for aspiration pneumonia? A prospective evaluation using a protected specimen brush and quantitative cultures. Intensive Care Med 1993;19:279-84. 10.1007/BF01690548 [PubMed] [Cross Ref]

203. Marik PE, Careau P. The role of anaerobes in patients with ventilatorassociated pneumonia and aspiration pneumonia: a prospective study. Chest 1999;115:178-83. 10.1378/chest.115.1.178 [PubMed][Cross Ref]

204. El-Solh AA, Pietrantoni $C$, Bhat $A$, et al. Microbiology of severe aspiration pneumonia in institutionalized elderly. Am J Respir Crit Care Med 2003;167:1650-4. 10.1164/rccm.200212-15430C[PubMed] [Cross Ref]

205. El-Solh AA, Vora H, Knight PR, 3rd, et al. Diagnostic use of serum procalcitonin levels in pulmonary aspiration syndromes. Crit Care Med 2011;39:1251-6. 10.1097/CCM.0b013e31820a942c [PMC free article] [PubMed] [Cross Ref]

206. Bartlett JG, Finegold SM. Anaerobic infections of the lung and pleural space. Am Rev Respir Dis1974;110:56-77. [PubMed]

207. Mendelson $\mathrm{CL}$. The aspiration of stomach contents into the lungs during obstetric anesthesia. Am J Obstet Gynecol 1946;52:191-205. 10.1016/ S0002-9378(16)39829-5 [PubMed] [Cross Ref]

208. Levison ME, Mangura CT, Lorber B, et al. Clindamycin compared with penicillin for the treatment of anaerobic lung abscess. Ann Intern Med 1983;98:466-71. 10.7326/0003-4819-98-4-466 [PubMed][Cross Ref] 
209. Gudiol F, Manresa F, Pallares R, et al. Clindamycin vs penicillin for anaerobic lung infections. High rate of penicillin failures associated with penicillin-resistant Bacteroides melaninogenicus. Arch Intern Med 1990;150:2525-9. 10.1001/ archinte.1990.00390230077010 [PubMed] [Cross Ref]

210. Centers for Disease Control and Prevention (CDC), Advisory Committee on Immunization PracticesUpdated recommendations for prevention of invasive pneumococcal disease among adults using the 23-valent pneumococcal polysaccharide vaccine (PPSV23). MMWR Morb Mortal Wkly Rep 2010;59:1102-6.[PubMed]

211. Centers for Disease Control and Prevention (CDC) Licensure of 13-valent pneumococcal conjugate vaccine for adults aged 50 years and older. MMWR Morb Mortal Wkly Rep 2012;61:394-5. [PubMed]

212. Pfizer laboratories (Pty) Ltd. Prevenar 13 suspension for injection. Final approved Package Insert - 26 November 2015.

213. Centers for Disease Control and Prevention (CDC) Use of 13-valent pneumococcal conjugate vaccine and 23-valent pneumococcal polysaccharide vaccine for adults with immunocompromising conditions: recommendations of the Advisory Committee on Immunization Practices (ACIP). MMWR Morb Mortal Wkly Rep 2012;61:816-9. [PubMed]
214. Tomczyk S, Bennett NM, Stoecker C, et al. Use of 13-valent pneumococcal conjugate vaccine and 23 -valent pneumococcal polysaccharide vaccine among adults aged $>/=65$ years: recommendations of the Advisory Committee on Immunization Practices (ACIP). MMWR Morb Mortal Wkly Rep 2014;63:822-5. [PMC free article] [PubMed]

215. French N, Gordon SB, Mwalukomo T, et al. A trial of a 7-valent pneumococcal conjugate vaccine in HIV-infected adults. N Engl J Med 2010;362:812-22. 10.1056/NEJMoa0903029 [PMC free article][PubMed] [Cross Ref]

216. Bonten MJ, Huijts SM, Bolkenbaas M, et al. Polysaccharide conjugate vaccine against pneumococcal pneumonia in adults. N Engl J Med 2015;372:1114-25. 10.1056/NEJMoa1408544 [PubMed] [Cross Ref]

217. DiazGranados CA , Dunning AJ, Kimmel M, et al. Efficacy of high-dose versus standard-dose influenza vaccine in older adults. N Engl J Med 2014;371:63545. 10.1056/NEJMoa1315727 [PubMed][Cross Ref]

218. Madhi SA, Maskew $M$, Koen $A$, et al. Trivalent inactivated influenza vaccine in African adults infected with human immunodeficient virus: double blind, randomized clinical trial of efficacy, immunogenicity, and safety. Clin Infect Dis 2011;52:128-37. 10.1093/cid/ciq004 [PubMed] [Cross Ref] 